\title{
MORLEY FINITE ELEMENT METHOD FOR THE VON KÁRMÁN OBSTACLE PROBLEM
}

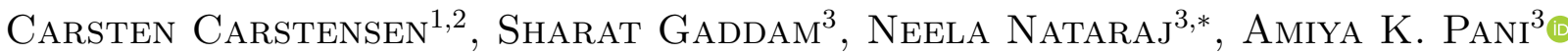 \\ AND DEVIKA SHYlaJA ${ }^{4}$
}

\begin{abstract}
This paper focusses on the von Kármán equations for the moderately large deformation of a very thin plate with the convex obstacle constraint leading to a coupled system of semilinear fourth-order obstacle problem and motivates its nonconforming Morley finite element approximation. The first part establishes the well-posedness of the von Kármán obstacle problem and also discusses the uniqueness of the solution under an a priori and an a posteriori smallness condition on the data. The second part of the article discusses the regularity result of Frehse from 1971 and combines it with the regularity of the solution on a polygonal domain. The third part of the article shows an a priori error estimate for optimal convergence rates for the Morley finite element approximation to the von Kármán obstacle problem for small data. The article concludes with numerical results that illustrates the requirement of smallness assumption on the data for optimal convergence rate.
\end{abstract}

Mathematics Subject Classification. 65K15, 65N12, 65N15, 65N30.

Received September 1, 2020. Accepted August 2, 2021.

\section{INTRODUCTION}

Short history of related work. The von Kármán equations [17] model the bending of very thin elastic plates through a system of fourth-order semi-linear elliptic equations; $c f .[2,17,23]$ and references therein for the existence of solutions, regularity, and bifurcation phenomena. The papers [5, 9, 12, 13, 25, 27,31,32] study the approximation and error bounds for regular solutions to von Kármán equations using conforming, mixed, hybrid, Morley, $C^{0}$ interior penalty and discontinuous Galerkin finite element methods (FEMs).

The obstacle problem is a prototypical example for a variational inequality and arises in contact mechanics, option pricing, and fluid flow problems. The location of the free boundary is not known a priori and forms a part of the solution procedure. For the theoretical and numerical aspects of variational inequalities, see [20,22]. A unified convergence analysis for the fourth-order linear two-sided obstacle problem of clamped Kirchhoff plates

Keywords and phrases. Obstacle problem, Morley FEM, von Karman equations, a priori estimate, regularity.

1 Department of Mathematics, HU Berlin, Berlin 10099, Germany.

2 Distinguished Visiting Professor, Department of Mathematics, IIT Bombay, Powai, Mumbai 400076, India.

3 Department of Mathematics, IIT Bombay, Powai, Mumbai 400076, India.

4 IITB-Monash Research Academy, IIT Bombay, Powai, Mumbai 400076, India.

*Corresponding author: nataraj.neela@gmail.com

(C) The authors. Published by EDP Sciences, SMAI 2021

This is an Open Access article distributed under the terms of the Creative Commons Attribution License (https://creativecommons.org/licenses/by/4.0), which permits unrestricted use, distribution, and reproduction in any medium, provided the original work is properly cited. 
in [6-8] studies $C^{1}$ FEMs, $C^{0}$ interior penalty methods, and classical nonconforming FEMs on convex domains and, analyse the $C^{0}$ interior penalty and the Morley FEM on polygonal domains.

The obstacle problem for von Kármán equations with a nonlinearity together with a free boundary offers additional difficulties. The obstacle problem in $[26,28,33]$ concerns a different plate model with continuation, spectral, and complementarity methods, while the papers $[29,30]$ study conforming penalty FEM.

The present paper is the first on the fourth-order semilinear obstacle problem of a (very thin) von Kármán plate. The article derives existence, uniqueness (under smallness assumption on data) and regularity results of the von Kármán obstacle problem. Nonconforming FEMs appear to be more attractive than the classical $C^{1}$ conforming FEMs, so this article suggests the Morley FEM to approximate the von Kármán obstacle problem and derives an optimal order a priori error estimate with the best approximation plus a linear perturbation.

Problem Formulation. Given an obstacle $\chi \in H^{2}(\Omega)$ with $\max \chi(\partial \Omega):=\max _{x \in \partial \Omega} \chi(x)<0$, define the non-empty, closed, and convex subset

$$
K:=\left\{\varphi \in H_{0}^{2}(\Omega): \varphi \geq \chi \text { a.e. in } \Omega\right\}
$$

of $H_{0}^{2}(\Omega)$ in a bounded polygonal domain $\Omega \subset \mathbb{R}^{2}$. The Hessian $D^{2}$ and von Kármán bracket $\left[\varphi_{1}, \varphi_{2}\right]:=$ $\varphi_{1 x x} \varphi_{2 y y}+\varphi_{1 y y} \varphi_{2 x x}-2 \varphi_{1 x y} \varphi_{2 x y}$ with partial derivatives $(\bullet)_{x y}:=\partial^{2}(\bullet) / \partial x \partial y$ etc. Define for $\varphi_{1}, \varphi_{2}, \varphi_{3} \in H_{0}^{2}(\Omega)$ the weak forms

$$
a\left(\varphi_{1}, \varphi_{2}\right):=\left(D^{2} \varphi_{1}, D^{2} \varphi_{2}\right)_{L^{2}(\Omega)} \text { and } b\left(\varphi_{1}, \varphi_{2}, \varphi_{3}\right):=-\frac{1}{2}\left(\left[\varphi_{1}, \varphi_{2}\right], \varphi_{3}\right)_{L^{2}(\Omega)}
$$

with the $L^{2}(\Omega)$ inner product $(\bullet, \bullet)_{L^{2}(\Omega)}$. It is well established [5, Corollary 2.3] and follows from symmetry of the von Kármán bracket $[\bullet, \bullet]$ that $b: H_{0}^{2}(\Omega)^{3} \rightarrow \mathbb{R}$ is symmetric with respect to all the three arguments. The weak formulation of the von Kármán obstacle problem seeks $(u, v) \in K \times H_{0}^{2}(\Omega)$ such that

$$
\begin{aligned}
& a\left(u, u-\varphi_{1}\right)+2 b\left(u, v, u-\varphi_{1}\right) \leq\left(f, u-\varphi_{1}\right)_{L^{2}(\Omega)} \quad \text { for all } \varphi_{1} \in K, \\
& a\left(v, \varphi_{2}\right)-b\left(u, u, \varphi_{2}\right)=0 \quad \text { for all } \varphi_{2} \in H_{0}^{2}(\Omega) .
\end{aligned}
$$

Results and overview. A smallness assumption on the data is derived in Section 2 to show that (1.2) is well-posed. The regularity results of Section 3 establish that any solution $(u, v)$ to $(1.2)$ satisfies $u, v \in H_{0}^{2}(\Omega) \cap$ $H^{2+\alpha}(\Omega) \cap C^{2}(\Omega)$ for the index $1 / 2<\alpha \leq 1$ with $\alpha=\min \left\{\alpha^{\prime}, 1\right\}$ and the index $\alpha^{\prime}$ of elliptic regularity [4] of the biharmonic operator in a polygonal domain $\Omega$. Section 4 introduces the Morley FEM and discusses the wellposedness of the discrete problem with an a priori and an a posteriori smallness condition on the data for global uniqueness. Section 5 derives a priori energy norm estimates of optimal order $\alpha$ for the Morley FEM under the smallness assumption on the data that guarantees global uniqueness of the minimizer on the continuous level. The article concludes with numerical results that illustrates the requirement of smallness assumption on the data for optimal convergence rate.

Notation. Standard notation on Lebesgue and Sobolev spaces and their norms apply throughout the paper. For $s>0$ and $1 \leq p \leq \infty$, let $|\bullet|_{s}$ and $\|\bullet\|_{s}$ (resp. $|\bullet|_{s, p}$ and $\left.\|\bullet\|_{s, p}\right)$ denote the semi-norm and norm on $H^{s}(\Omega)$ (resp. $\left.W^{s, p}(\Omega)\right) ;\|\bullet\|_{-s}$ denotes the norm in $H^{-s}(\Omega)$. The standard $L^{2}$ inner product and norm are denoted by $(\bullet, \bullet)_{L^{2}(\Omega)}$ and $\|\bullet\|_{L^{2}(\Omega)}$. The triple norm $\|\bullet\|:=\left\|D^{2} \bullet\right\|_{L^{2}(\Omega)}$ is the energy norm defined by the Hessian and $\|\bullet\|_{\mathrm{pw}}:=\left\|D_{\mathrm{pw}}^{2} \bullet\right\|_{L^{2}(\Omega)}$ is its piecewise version with the piecewise Hessian $D_{\mathrm{pw}}^{2},[\bullet, \bullet]_{\mathrm{pw}}$ denotes the piecewise version of the von Kármán bracket $[\bullet, \bullet]$ with respect to an underlying (non-displayed) triangulation. $H^{-2}(\Omega)$ is the dual space of the Hilbert space $\left(H_{0}^{2}(\Omega),\|\bullet\|\right)$. The elliptic regularity index $1 / 2<\alpha \leq 1$ is determined by the interior angles of the domain $\Omega[4]$ and is the same throughout this paper. The notation $A \lesssim B$ abbreviates $A \leq \mathrm{CB}$ for some positive generic constant $C$ which depends on $\|u\|,\|v\|,\|u\|_{2+\alpha},\|v\|_{2+\alpha},\|f\|_{L^{2}(\Omega)} ; A \approx B$ abbreviates $A \lesssim B \lesssim A$. 


\section{WELL-POSEDNESS}

This section establishes the well-posedness of the problem (1.2). The existence of a solution to (1.2) follows with the direct method in the calculus of variations. The subsequent bound applies often in this paper and is based on Sobolev embedding. Let $C_{\mathrm{S}}$ denote the Sobolev constant in the Sobolev embedding $H_{0}^{2}(\Omega) \hookrightarrow C(\bar{\Omega})$ and let $C_{\mathrm{F}}$ denote the Friedrichs constant with

$$
\|v\|_{L^{\infty}(\Omega)} \leq C_{\mathrm{S}}\|v\|_{H_{0}^{2}(\Omega)} \quad \text { and } \quad\|v\|_{L^{2}(\Omega)} \leq C_{\mathrm{F}}\|v\| \text { for all } v \in H_{0}^{2}(\Omega) .
$$

Lemma 2.1 (bound for $b(\bullet, \bullet, \bullet)[9])$. The trilinear form $b(\bullet, \bullet, \bullet)$ from $(1.1)$ satisfies, for all $\varphi_{1}, \varphi_{2}, \varphi_{3} \in$ $H_{0}^{2}(\Omega)$, that $b\left(\varphi_{1}, \varphi_{2}, \varphi_{3}\right) \leq\left\|\varphi_{1}\right\|\left\|\varphi_{2}\right\|\left\|\varphi_{3}\right\|_{L^{\infty}(\Omega)} \leq C_{\mathrm{S}}\left\|\varphi_{1}\right\|\left\|\varphi_{2}\right\|\left\|\varphi_{3}\right\| \|$.

For all $\xi \in H_{0}^{2}(\Omega)$, Lemma 2.1 implies $b(\xi, \xi, \bullet) \in H^{-2}(\Omega)$. Define $G: H_{0}^{2}(\Omega) \rightarrow H_{0}^{2}(\Omega)$ by

$$
a(G(\varphi), \psi)=b(\varphi, \varphi, \psi) \text { for all } \varphi, \psi \in H_{0}^{2}(\Omega) .
$$

This means $G(\xi)$ is the Riesz representation of the linear bounded functional $b(\xi, \xi, \bullet)$ in the Hilbert space $\left(H_{0}^{2}(\Omega), a(\bullet, \bullet)\right)$. Consider the minimizer $u$ of the functional $j(\xi)$ for $\xi \in K$ and

$$
j(\xi):=\frac{1}{2}\|\xi\|^{2}+\frac{1}{2}\|G(\xi)\|^{2}-(f, \xi)_{L^{2}(\Omega)} .
$$

The equivalence of (1.2) with (2.3), for $K=H_{0}^{2}(\Omega)$, is established in [17, Thm. 5.8.3]. Analogous arguments also establish the equivalence, for any non-empty, closed, and convex subset $K$ of $H_{0}^{2}(\Omega)$, so the proof is omitted. This implies that, to prove the existence of a solution to (1.2), it is sufficient to prove the existence of a minimizer to $(2.3)$.

Theorem 2.2 (existence). Given $(f, \chi) \in L^{2}(\Omega) \times H^{2}(\Omega)$ with $\max \chi(\partial \Omega)<0$, there exists a minimizer of $j(\bullet)$ in $K$; each minimizer $u$ and $v:=G(u)$ solve $(1.2)$.

Proof. Given $\xi \in K$, the definition of $j(\bullet)$ in (2.3) and the Cauchy-Schwarz inequality lead to

$$
\|\xi\|^{2}+\|G(\xi)\|^{2}-2\|f\|_{-2}\|\xi\| \leq 2 j(\xi) .
$$

This implies the lower bound

$$
-\infty<-\|f\|_{-2}^{2}=\min _{t \geq 0}\left(t^{2}-2 t\|f\|_{-2}\right) \leq 2 j(\xi) \text { for all } \xi \in K .
$$

Consequently, there exists a sequence $\left(u_{n}\right)_{n \in \mathbb{N}}$ in $K$ such that

$$
j\left(u_{n}\right) \rightarrow \beta:=\inf _{\xi \in K} j(\xi) \in \mathbb{R} .
$$

The Cauchy-Schwarz and the Young inequalities lead to

$$
\left\|u_{n}\right\|^{2}+2\left\|G\left(u_{n}\right)\right\|^{2}-4\|f\|_{-2}^{2} \leq 2\left\|u_{n}\right\|^{2}+2\left\|G\left(u_{n}\right)\right\|^{2}-4\|f\|_{-2}\left\|u_{n}\right\| \leq 4 j\left(u_{n}\right) .
$$

Consequently, $\left\|u_{n}\right\|^{2}+2\left\|G\left(u_{n}\right)\right\|^{2} \leq 4 j\left(u_{n}\right)+4\|f\|_{-2}^{2}$. Since $j\left(u_{n}\right)$ is convergent, the sequences $\left(u_{n}\right)_{n \in \mathbb{N}}$ and $\left(G\left(u_{n}\right)\right)_{n \in \mathbb{N}}$ are bounded in $H_{0}^{2}(\Omega)$. Hence, there exist $u, w \in H_{0}^{2}(\Omega)$ and a weakly convergent subsequence $\left(u_{n_{k}}\right)_{k \in \mathbb{N}}$ such that

$$
u_{n_{k}} \rightarrow u \text { and } G\left(u_{n_{k}}\right) \rightarrow w \text { weakly in } H_{0}^{2}(\Omega) \text { as } k \rightarrow \infty .
$$

The non-empty closed convex set $K$ of $H_{0}^{2}(\Omega)$ is sequentially weakly closed and so $u \in K$. Since $u_{n_{k}}$ converges weakly to $u$ in $H_{0}^{2}(\Omega)$, this implies

$$
\int_{\Omega} f u_{n_{k}} \rightarrow \int_{\Omega} f u \text { as } k \rightarrow \infty .
$$


The compact embedding of $H_{0}^{2}(\Omega)$ in $L^{2}(\Omega)$ implies $u_{n_{k}} \rightarrow u$ in $L^{2}(\Omega)$. Further for a given $\varphi \in \mathcal{D}(\Omega)$, the definition of $G(\bullet)$ in $(2.2)$, the symmetry of $b(\bullet, \bullet, \bullet)$ with respect to second and third arguments, and the weak convergence of $u_{n_{k}} \rightarrow u$ in $H_{0}^{2}(\Omega)$ lead to

$$
a\left(G\left(u_{n_{k}}\right), \varphi\right)=b\left(u_{n_{k}}, \varphi, u_{n_{k}}\right) \rightarrow b(u, \varphi, u)=a(G(u), \varphi) \text { as } k \rightarrow \infty .
$$

Since $\varphi$ is arbitrary in the dense set $\mathcal{D}(\Omega)$ of $H_{0}^{2}(\Omega)$, this means $G\left(u_{n_{k}}\right) \rightarrow G(u)$ weakly in $H_{0}^{2}(\Omega)$ as $k \rightarrow \infty$.

The sequentially weak lower semi-continuity of the norm $\|\bullet\|$ shows $j(u) \leq \liminf k_{k} j\left(u_{n_{k}}\right)$. This and $\lim _{k \rightarrow \infty} j\left(u_{n_{k}}\right)=\beta \leq \liminf _{k} j\left(u_{n_{k}}\right)$ prove that $u$ minimizes $j$ in $K$. By the definition of $G(\bullet)$ in $(2.2),(u, G(u))$ solves (1.2). This concludes the proof.

Theorem 2.3 establishes an a priori bound and the uniqueness of the solution to (1.2). Recall the Sobolev (resp. Friedrichs) constant $C_{\mathrm{S}}$ (resp. $C_{\mathrm{F}}$ ) from (2.1).

Theorem 2.3 (a priori bound and uniqueness). Given $(f, \chi) \in L^{2}(\Omega) \times H^{2}(\Omega)$ with $\max \chi(\partial \Omega)<0$, there exists a positive constant $C(\chi)$ that depends only on $\chi$ such that any solution $(u, v)$ to $(1.2)$ satisfies $(a)-(b)$.

(a) $\frac{1}{2}\|u\|^{2}+\|v\|^{2} \leq N^{2}(f, u):=2 j(u)+2 C_{\mathrm{F}}^{2}\|f\|^{2} \leq M^{2}(f, \chi):=C(\chi)+3 C_{\mathrm{F}}^{2}\|f\|_{L^{2}(\Omega)}^{2}$.

(b) If $\frac{C_{S}^{2}}{4}\|u\|^{2}+C_{S}\|v\|<\frac{1}{2}$, then $(u, v)$ is the only solution to (1.2).

Proof of (a). Since $u$ is the minimizer of (2.3), the Young inequality implies, for any $\varphi \in K$, that

$$
\|u\|^{2}+\|G(u)\|^{2} \leq 2 j(\varphi)+2(f, u)_{L^{2}(\Omega)} \leq 2 j(\varphi)+2 C_{\mathrm{F}}^{2}\|f\|_{L^{2}(\Omega)}^{2}+\frac{1}{2}\|u\|^{2} .
$$

This proves for the minimizer $u$ of $j(\bullet)$ that

$$
\frac{1}{2}\|u\|^{2}+\|G(u)\|^{2} \leq 2 j(u)+2 C_{\mathrm{F}}^{2}\|f\|_{L^{2}(\Omega)}^{2}:=N^{2}(f, u) \leq N^{2}(f, \varphi) .
$$

Since $\max \chi(\partial \Omega)<0,\{\chi \geq 0\}:=\{x \in \Omega: \chi(x) \geq 0\}$ is a compact subset of $\Omega$ and there exists an open set $\Omega_{+}$around $\{\chi \geq 0\}$ such that $\overline{\Omega_{+}}$is a compact subset of $\Omega$. Consider the cut-off function $\psi \in \mathcal{D}(\Omega)$ such that $0 \leq \psi \leq 1, \psi=1$ in $\{\chi \geq 0\}, \operatorname{supp}(\psi) \subset \Omega_{+}$, and define $\varphi:=\chi \psi \in H_{0}^{2}(\Omega)$. Then, $\varphi \geq \chi$ in $\Omega$, and so $\varphi \in K$. The construction of $\varphi$ ensures that

$$
\|\varphi\|=|\varphi|_{H^{2}\left(\Omega_{+}\right)} \leq C(\psi)\|\chi\|_{H^{2}\left(\Omega_{+}\right)} .
$$

This inequality, the definition of $G(\bullet)$, and Lemma 2.1 lead to

$$
\|G(\varphi)\|^{2}=b(\varphi, \varphi, G(\varphi)) \leq C_{\mathrm{S}}\|\varphi\|^{2}\|G(\varphi)\| \leq C_{\mathrm{S}} C^{2}(\psi)\|G(\varphi)\|\|\chi\|_{H^{2}\left(\Omega_{+}\right)}^{2} .
$$

Consequently, $\|G(\varphi)\| \leq C_{S} C^{2}(\psi)\|\chi\|_{H^{2}\left(\Omega_{+}\right)}^{2}$. An application of the bounds for $\varphi$ and $G(\varphi)$ in $(2.5)$ concludes the proof of final estimate of part $(a)$ with $C(\chi):=2 C^{2}(\psi)\|\chi\|_{H^{2}\left(\Omega_{+}\right)}^{2}+C_{\mathrm{S}}^{2} C^{4}(\psi)\|\chi\|_{H^{2}\left(\Omega_{+}\right)}^{4}$ and $u$ being the minimizer of $j(\bullet)$ implies $N(f, u) \leq N(f, \psi \chi) \leq M(f, \chi)$.

Proof of $(b)$. Recall the definition of $G(\bullet)$ from $(2.2)$ and let $\left(u_{1}, G\left(u_{1}\right)\right)$ and $\left(u_{2}, G\left(u_{2}\right)\right)$ be two solutions to (1.2). Set $e=u_{1}-u_{2}, \delta=G\left(u_{1}\right)-G\left(u_{2}\right)$, and choose $u=u_{1}, \varphi_{1}=u_{2}$ (respectively, $\left.u=u_{2}, \varphi_{1}=u_{1}\right)$ in (1.2a) and add the resulting inequalities to deduce that

$$
\frac{1}{2}\|e\|^{2} \leq-b\left(u_{1}, G\left(u_{1}\right), e\right)+b\left(u_{2}, G\left(u_{2}\right), e\right)=-b\left(e, e, G\left(u_{1}\right)\right)-b\left(e, \delta, u_{2}\right) .
$$

Elementary algebra with $(1.2 \mathrm{~b})$, the definition of $G(\bullet)$ and symmetry of $b(\bullet, \bullet, \bullet)$ with respect to the three variables show

$$
\|\delta\|^{2}=b\left(u_{1}, u_{1}, \delta\right)-b\left(u_{2}, u_{2}, \delta\right)=b\left(e, \delta, u_{1}\right)+b\left(e, \delta, u_{2}\right)
$$


The combination of (2.7)-(2.8) and Lemma 2.1 lead to

$$
\frac{1}{2}\|e\|^{2}+\|\delta\|^{2} \leq b\left(e, \delta, u_{1}\right)-b\left(e, e, G\left(u_{1}\right)\right) \leq C_{\mathrm{S}}\|e\|\|\| \delta\|\| u_{1}\left\|+C_{\mathrm{S}}\right\| e\left\|^{2}\right\| G\left(u_{1}\right) \| .
$$

If $\|\delta\|=0<\|e\|$, then $(2.9)$ implies $\frac{1}{2} \leq C_{S}\left\|G\left(u_{1}\right)\right\|$; a contradiction to the smallness assumption $\frac{C_{S}^{2}}{4}\left\|u_{1}\right\|^{2}+$ $C_{S}\left\|G\left(u_{1}\right)\right\|<\frac{1}{2}$. If $\|\delta\|>0$, then $\|e\|:=t\|\delta\|$ for some real $t \geq 0$, and (2.9) is equivalent to

$0 \leq\left(t-\frac{C_{S}}{2}\left\|u_{1}\right\|\right)^{2} \leq \frac{C_{S}^{2}}{4}\left\|u_{1}\right\|^{2}+C_{S}\left\|G\left(u_{1}\right)\right\|-\frac{1}{2}<0$.

This contradiction proves $\|e\|=0=\|\delta\|$.

Remark 2.4 (a priori and a posteriori criteria for uniqueness). The a priori smallness assumption on the data $C_{\mathrm{S}} M(f, \chi)<1 / 2$ and the a posteriori criterion $C_{\mathrm{S}} N(f, \varphi)<1 / 2$ for some $\varphi \in K$ imply $C_{\mathrm{S}} N(f, u)<$ $1 / 2$ and so uniqueness of the solution to (1.2). For a proof, abbreviate $x^{2}:=\frac{1}{2}\|u\|^{2}, y:=\|v\|$, and $N:=$ $N(f, u)$ so that Theorem 2.3a reads $x^{2}+y^{2} \leq N^{2}$. Then $\frac{C_{S}^{2}}{4}\|u\|^{2}+C_{S}\|v\| \leq \frac{C_{S}^{2}}{2} x^{2}+C_{S} y \leq \max _{\xi^{2}+\eta^{2} \leq N^{2}}\left(\frac{C_{S}^{2}}{2} \xi^{2}+\right.$ $\left.C_{S} \eta\right)=\max _{0 \leq \eta \leq N}\left(\frac{C_{S}^{2}}{2}\left(N^{2}-\eta^{2}\right)+C_{S} \eta\right)$. The real function $\frac{C_{S}^{2}}{2}\left(N^{2}-\eta^{2}\right)+C_{S} \eta$ is monotonically increasing in $\eta$ for $0 \leq \eta \leq N$ and hence the aforementioned maximum is equal to $C_{S} N<1 / 2$. This and Theorem 2.3a conclude the proof.

\section{REgularity}

The regularity result in [18] will be employed for modified obstacles in the biharmonic obstacle problem. Given any obstacle $\tilde{\chi} \in H^{2}(\Omega) \cap H_{\text {loc }}^{3}(\Omega)$ with $\max \tilde{\chi}(\partial \Omega)<0$, define a corresponding non-empty, closed and convex subset $K(\widetilde{\chi}):=\left\{\varphi \in H_{0}^{2}(\Omega): \varphi \geq \tilde{\chi}\right.$ a.e. in $\left.\Omega\right\}$ of $H_{0}^{2}(\Omega)$ and notice $K=K(\chi)$ for the original obstacle $\chi$ from (1.2). Given any such $\tilde{\chi}$, and $f \in L^{2}(\Omega)$, consider the problem that seeks the solution $\phi \in K(\widetilde{\chi})$ to

$$
a(\phi, \phi-\psi) \leq(f, \phi-\psi)_{L^{2}(\Omega)} \quad \text { for all } \psi \in K(\widetilde{\chi}) .
$$

Theorem 3.1 (Frehse 1971). Let $\Omega$ be an open bounded connected subset of $\mathbb{R}^{2}$. If $\phi \in K(\widetilde{\chi})$ solves (3.1) for $\tilde{\chi} \in H^{2}(\Omega) \cap H_{\mathrm{loc}}^{3}(\Omega)$ with $\max \widetilde{\chi}(\partial \Omega)<0$, then $\phi \in H_{0}^{2}(\Omega) \cap H_{\mathrm{loc}}^{3}(\Omega)$.

Proof. Frehse's result [18, Thm. 1] shows $\phi \in H_{0}^{2}(\Omega) \cap H_{\text {loc }}^{3}(\Omega)$ even under the much more involved assumption $\tilde{\chi} \in H^{3}(\Omega)$ and $\max \tilde{\chi}(\partial \Omega) \leq 0$. The theorem at hand assures that $\max \tilde{\chi}(\partial \Omega)<0$ and the proof will establish that Frehse's result can be adapted. The remaining parts of this proof establish that for an appropriate $\hat{\chi} \in$ $H^{3}(\Omega)$ constructed in the sequel, $\phi$ satisfies (3.1) with an obstacle $\widehat{\chi}$. Since $\max \widetilde{\chi}(\partial \Omega)<0$ and $\phi \in H_{0}^{2}(\Omega)$, there exist $\epsilon>0$ and $\delta<0$ such that $\tilde{\chi}<\delta<\phi$ in $\overline{N(2 \epsilon, \partial \Omega)}$, where $N(2 \epsilon, \partial \Omega):=\{x \in \Omega: \operatorname{dist}(x, \partial \Omega)<2 \epsilon\}$. Select cut-off functions $0 \leq \psi_{1}, \psi_{2} \in C^{\infty}(\bar{\Omega})$ such that $\psi_{1}+\psi_{2} \equiv 1$ in $\bar{\Omega}$ and

$$
\psi_{1}=\left\{\begin{array}{ll}
1 & \text { in } \overline{N(\epsilon, \partial \Omega)}, \\
0 & \text { in } \Omega \backslash N(2 \epsilon, \partial \Omega)
\end{array} \quad \text { and } \quad \psi_{2}= \begin{cases}0 & \text { in } \overline{N(\epsilon, \partial \Omega)}, \\
1 & \text { in } \Omega \backslash N(2 \epsilon, \partial \Omega) .\end{cases}\right.
$$

Consider $\widehat{\chi}:=\delta \psi_{1}+\widetilde{\chi} \psi_{2}$ and derive the following three inequalities

$$
\begin{gathered}
\tilde{\chi}(x)<\delta=\widehat{\chi}(x)<\phi(x) \text { for all } x \in \overline{N(\epsilon, \partial \Omega)}, \\
\widetilde{\chi}(x)=\widehat{\chi}(x) \leq \phi(x) \text { for all } x \in \Omega \backslash N(2 \epsilon, \partial \Omega), \\
\widetilde{\chi}(x)<\delta \psi_{1}(x)+\widetilde{\chi}(x) \psi_{2}(x)=\widehat{\chi}(x)<\delta<\phi(x) \text { for all } x \in(\Omega \backslash \overline{N(\epsilon, \partial \Omega)}) \cap N(2 \epsilon, \partial \Omega) .
\end{gathered}
$$


The above three inequalities imply $\tilde{\chi} \leq \hat{\chi} \leq \phi$ in $\bar{\Omega}$ and $\tilde{\chi} \in H^{3}(\Omega \backslash N(\epsilon, \partial \Omega))$. By construction, $\hat{\chi}$ is the combination of a $H^{3}(\Omega)$ and a $C^{\infty}(\bar{\Omega})$ function, and hence, $\hat{\chi} \in H^{3}(\Omega)$. Given $\hat{\chi}$ as an obstacle, the solution $\phi \in K(\widehat{\chi})$ to $(3.1)$ also satisfies

$$
a(\phi, \phi-\psi) \leq(f, \phi-\psi)_{L^{2}(\Omega)} \quad \text { for all } \psi \in K(\widehat{\chi}) .
$$

Since the obstacle $\widehat{\chi}$ of the problem (3.3) belongs to $H_{0}^{2}(\Omega) \cap H^{3}(\Omega)$, [18, Thm. 1] proves $\phi \in H_{\text {loc }}^{3}(\Omega)$.

The final regularity result of the von Kármán obstacle problem relies on the following three lemmas.

Lemma 3.2 ([3, Equation (2.6)], [4, Thm. 2]). Let $\Omega$ be a bounded polygonal domain in $\mathbb{R}^{2}$. If $w \in H_{0}^{2}(\Omega)$ solves the biharmonic problem, $a(w, \varphi)=f(\varphi)$ for all $\varphi \in H_{0}^{2}(\Omega)$, with data $f \in H^{-1}(\Omega)$ (resp. $L^{2}(\Omega)$ ), then $w \in H_{\mathrm{loc}}^{3}(\Omega) \cap H^{2+\alpha}(\Omega)$ (resp. $H_{\mathrm{loc}}^{4}(\Omega)$ ). If the bounded Lipschitz domain $\Omega$ has a $C^{2+\gamma}$ boundary for some $0<\gamma<1$ and $f \in L^{2}(\Omega)$ (resp. $H^{-1}(\Omega)$ ), then the solution $w$ belongs to $H^{4}(\Omega)$ (resp. $H^{3}(\Omega)$ ).

Lemma 3.3 ([4, Thm. 7$])$. Let $\Omega$ be a bounded polygonal domain in $\mathbb{R}^{2}$. If $\left(w_{1}, w_{2}\right) \in H_{0}^{2}(\Omega) \times H_{0}^{2}(\Omega)$ is a solution to the von Kármán equations, $a\left(w_{1}, \varphi_{1}\right)+2 b\left(w_{1}, w_{2}, \varphi_{1}\right)+a\left(w_{2}, \varphi_{2}\right)-b\left(w_{1}, w_{1}, \varphi_{2}\right)=f\left(\varphi_{1}\right)$ for all $\left(\varphi_{1}, \varphi_{2}\right) \in H_{0}^{2}(\Omega) \times H_{0}^{2}(\Omega)$, with data $f \in H^{-1}(\Omega)$, then $\left(w_{1}, w_{2}\right) \in H^{2+\alpha}(\Omega) \times H^{2+\alpha}(\Omega)$.

The remaining parts of this section return to (1.2) with $f \in L^{2}(\Omega)$ and a polygonal domain $\Omega$.

Lemma 3.4. If $(u, v) \in K \times H_{0}^{2}(\Omega)$ solves (1.2) for $\chi \in H^{2}(\Omega)$ with $\max \chi(\partial \Omega)<0$, then $[u, v] \in H^{-1}(\Omega)$.

Proof. The Sobolev embedding $H^{1+\epsilon}(\Omega) \hookrightarrow L^{\infty}(\Omega)$ and $u \in H_{0}^{2}(\Omega)$ imply $[u, u] \in H^{-1-\epsilon}(\Omega)$ for any $\epsilon>0$. A shift theorem [1, Thm. 8] in (1.2b) shows $v \in H^{2+\alpha-\epsilon}(\Omega)$ for $1 / 2<\alpha \leq 1$. Given $\alpha$, choose $\epsilon$ such that $\alpha-\epsilon>1 / 2$. Then, [5, Lemma 2.2] implies

$$
([u, v], \varphi)_{L^{2}(\Omega)}=-\int_{\Omega} \operatorname{cof}\left(D^{2} v\right) \nabla u \cdot \nabla \varphi \mathrm{dx} \leq\left\|\operatorname{cof}\left(D^{2} v\right)\right\|_{L^{4}(\Omega)}\|\nabla u\|_{L^{4}(\Omega)}\|\nabla \varphi\|_{L^{2}(\Omega)}
$$

for all $\varphi \in H_{0}^{1}(\Omega)$. This and the Sobolev embeddings $H^{2}(\Omega) \hookrightarrow W^{1,4}(\Omega), H^{2+\alpha-\epsilon}(\Omega) \hookrightarrow W^{2,4}(\Omega)$ conclude the proof.

Theorem 3.5 (regularity for von Kármán obstacle problem). Let $\Omega$ be a bounded polygonal domain in $\mathbb{R}^{2}$. If $(u, v) \in K \times H_{0}^{2}(\Omega)$ solves (1.2) for $\chi \in H^{2}(\Omega) \cap H_{\mathrm{loc}}^{3}(\Omega) \cap C^{2}(\Omega)$ with $\max \chi(\partial \Omega)<0$, then $u, v \in$ $H^{2+\alpha}(\Omega) \cap H_{\mathrm{loc}}^{3}(\Omega) \cap C^{2}(\Omega)$.

Proof. Let $(u, v)$ solve $(1.2)$ and let $w \in K(\chi)$ solve

$$
a(w, w-\varphi) \leq(f+[u, v], w-\varphi)_{L^{2}(\Omega)} \quad \text { for all } \varphi \in K(\chi) .
$$

Let $w_{1} \in H_{0}^{2}(\Omega)$ be the Riesz representation of $-[u, v]$ in the Hilbert space $\left(H_{0}^{2}(\Omega), a(\bullet, \bullet)\right)$, i.e., $w_{1}$ satisfies $a\left(w_{1}, \varphi_{1}\right)=-\left([u, v], \varphi_{1}\right)_{L^{2}(\Omega)}$ for all $\varphi_{1} \in H_{0}^{2}(\Omega)$. Lemmas 3.4 and 3.2 show that $w_{1} \in H_{\mathrm{loc}}^{3}(\Omega) \cap H^{2+\alpha}(\Omega)$. Translate the obstacle $\chi$ of (3.4) to $\chi+w_{1}$ and set $\widetilde{w}:=w+w_{1}$ to obtain

$$
a(\widetilde{w}, \widetilde{w}-\widetilde{\varphi}) \leq(f, \widetilde{w}-\widetilde{\varphi})_{L^{2}(\Omega)} \quad \text { for all } \widetilde{\varphi} \in K\left(\chi+w_{1}\right) .
$$

Since the obstacle $\chi+w_{1} \in H_{\mathrm{loc}}^{3}(\Omega)$, Theorem 3.1 implies $\widetilde{w} \in H_{\mathrm{loc}}^{3}(\Omega)$. Also $w_{1} \in H_{\mathrm{loc}}^{3}(\Omega)$ implies $w=\widetilde{w}-w_{1} \in H_{\mathrm{loc}}^{3}(\Omega)$. The solution $u$ to (1.2) also solves (3.4). The uniqueness of the solution in (3.1) implies that $u \in H_{\mathrm{loc}}^{3}(\Omega)$.

Let the contact region $\mathcal{C}:=\{x \in \Omega: u(x)=\chi(x)\}$. Define a cut-off function $\xi \in C^{\infty}(\bar{\Omega})$ with $\xi \equiv 1$ in $N(\epsilon, \partial \Omega)$ for some $\epsilon>0$ such that $\overline{N(2 \epsilon, \partial \Omega)} \cap \mathcal{C}=\emptyset$, i.e., $\operatorname{supp}(\xi) \subset N(2 \epsilon, \partial \Omega)$ keeps a positive distance to $\mathcal{C}$. The strong form of $(1.2 \mathrm{~b})$ and elementary manipulations show

$$
\Delta^{2} v=-\frac{1}{2}[u, u]=-\frac{1}{2}[\xi u, \xi u]-[(1-\xi) u, \xi u]-\frac{1}{2}[(1-\xi) u,(1-\xi) u] .
$$


Let $v_{1} \in H_{0}^{2}(\Omega)$ solve $\Delta^{2} v_{1}=f_{1}$ for $f_{1}:=-[(1-\xi) u, \xi u]-\frac{1}{2}[(1-\xi) u,(1-\xi) u]$. Since $u \in H_{0}^{2}(\Omega) \cap H_{\text {loc }}^{3}(\Omega)$, $\xi u \in H_{0}^{2}(\Omega),(1-\xi) u \in H^{3}(\Omega)$ and $f_{1} \in H^{-1}(\Omega)$. Lemma 3.2 leads to $v_{1} \in H^{2+\alpha}(\Omega)$. Also, $\Delta^{2}\left(v-v_{1}\right)=-\frac{1}{2}[\xi u, \xi u]$ in $\Omega$. Since $\operatorname{supp}(\xi) \cap \mathcal{C}=\emptyset,(1.2 \mathrm{a})$ implies $\Delta^{2} u=f+[u, v]$ in $\operatorname{supp}(\xi)$. Since $(1-\xi) u \in H^{3}(\Omega)$ and $v_{1} \in H^{2+\alpha}(\Omega)$, it follows from the arguments in Lemma 3.4 that $f_{2}:=f+\left[\xi u, v_{1}\right]+[(1-\xi) u, v]-\Delta^{2}((1-\xi) u) \in H^{-1}(\Omega)$. This and elementary manipulations lead to

$$
\begin{aligned}
\Delta^{2}(\xi u) & =\Delta^{2} u-\Delta^{2}((1-\xi) u)=f+[\xi u, v]+[(1-\xi) u, v]-\Delta^{2}((1-\xi) u) \\
& =f+\left[\xi u, v-v_{1}\right]+\left[\xi u, v_{1}\right]+[(1-\xi) u, v]-\Delta^{2}((1-\xi) u)=f_{2}+\left[\xi u, v-v_{1}\right]
\end{aligned}
$$

In other words, $\left(\xi u, v-v_{1}\right)$ solves the von Kármán equations for the right-hand side $f_{2} \in H^{-1}(\Omega)$ and $\xi u \in$ $H^{2+\alpha}(\Omega)$. Since $\xi u,(1-\xi) u \in H^{2+\alpha}(\Omega)$, it follows $u=\xi u+(1-\xi) u \in H^{2+\alpha}(\Omega)$. Return to the proof of Lemma 3.4 with the improved regularity $u \in H^{2+\alpha}(\Omega)$ to deduce that $[u, u] \in H^{-1}(\Omega)$. Since $v=G(u)$ solves (1.2b), this shows $v \in H^{2+\alpha}(\Omega) \cap H_{\mathrm{loc}}^{3}(\Omega)$.

The above arguments imply $u, v \in H^{2+\alpha}(\Omega)$, for $\alpha>1 / 2$, and the Sobolev embedding $H^{2+\alpha}(\Omega) \hookrightarrow W^{2,4}(\Omega)$ shows $[u, u],[u, v] \in L^{2}(\Omega)$. By Lemma 3.2 , the solution to $\Delta^{2} v=-\frac{1}{2}[u, u]$ belongs to $H_{\text {loc }}^{4}(\Omega)$. Then, the continuous Sobolev embedding $H_{\mathrm{loc}}^{4}(\Omega) \hookrightarrow C^{2}(\Omega)$ implies $v \in C^{2}(\Omega)$. Since $u \in H_{0}^{2}(\Omega), \chi \in C^{2}(\Omega)$, and $\max \chi(\partial \Omega)<0$, the arguments in the proof of Theorem 3.1 lead to $\tilde{\chi} \in C^{2}(\bar{\Omega})$ such that $\chi \leq \tilde{\chi} \leq u$. This shows that $u \in K(\widetilde{\chi})$, and hence with $\tilde{f}:=f+[u, v] \in L^{2}(\Omega), u$ solves

$$
a(u, u-\varphi) \leq(\tilde{f}, u-\varphi)_{L^{2}(\Omega)} \quad \text { for all } \varphi \in K(\widetilde{\chi}) .
$$

[8, Appendix A] establishes the regularity result for the biharmonic obstacle problem (3.7), which implies that the solution $u$ belongs to $C^{2}(\Omega)$. This concludes the proof.

Remark 3.6 (alternative proof). Lemma 3.4 and Theorem 3.1 prove $u \in H_{\mathrm{loc}}^{3}(\Omega)$. Since $u=w$ satisfies the biharmonic equation near $\partial \Omega$ in the sense of (3.6), $u \in H^{2+\alpha}(N(\epsilon, \partial \Omega))$. This and $u \in H_{\text {loc }}^{3}(\Omega)$ imply $u \in H^{2+\alpha}(\Omega)$. Since $u \in L_{\mathrm{loc}}^{4}(\Omega),[u, u] \in L_{\mathrm{loc}}^{2}(\Omega)$ and so $v \in H_{\mathrm{loc}}^{4}(\Omega)$ follows from interior regularity. Altogether, $f+[u, v] \in L_{\text {loc }}^{2}(\Omega)$ and transferring (3.4) to a biharmonic obstacle problem with zero forcing, we obtain $u \in C^{2}(\Omega)$ from $[10$, Sect. 6$]$ and $\chi \in C^{2}(\Omega)$.

Remark 3.7 $\left(C^{2}(\bar{\Omega})\right.$ regularity). If the bounded Lipschitz domain $\Omega$ has a $C^{2+\gamma}$ boundary for some $0<\gamma<1$, then any solution $(u, v)$ to $(1.2)$ belongs to $C^{2}(\bar{\Omega}) \times C^{2}(\bar{\Omega})$. In fact, $[u, u] \in L^{2}(\Omega)$, Lemma 3.2 , and continuous Sobolev embedding $H^{4}(\Omega) \hookrightarrow C^{2}(\bar{\Omega})$ imply that the solution $v$ to $(1.2 \mathrm{~b})$ belongs to $C^{2}(\bar{\Omega})$. An application of Lemma 3.2 to the arguments of $[8$, Appendix A] for (3.7) conclude that the solution $u$ to (1.2a) belongs to $C^{2}(\bar{\Omega})$.

\section{Morley finite Element approximation}

The first subsection discusses some preliminaries on the Morley FEM and interpolation and enrichment operators. The second subsection derives the existence, uniqueness under a computable smallness assumption and an a priori bound of the discrete solution.

\subsection{Preliminaries}

Let $\mathcal{T}$ be an admissible and regular triangulation of the polygonal bounded Lipschitz domain $\Omega$ into triangles in $\mathbb{R}^{2}$, let $h_{T}$ be the diameter of a triangle $T \in \mathcal{T}$ and $h_{\max }:=\max _{T \in \mathcal{T}} h_{T}$. For any $\epsilon>0$, let $\mathbb{T}(\epsilon)$ denote the set of all triangulations $\mathcal{T}$ with $h_{\max }<\epsilon$. For a non-negative integer $m$, let $\mathcal{P}_{m}(\mathcal{T})$ denote the space of piecewise polynomials of degree at most $m$. Let $\Pi_{0}$ denote the $L^{2}$ projection onto the space $\mathcal{P}_{0}(\mathcal{T})$ of piecewise constants and let $\mathcal{E}$ and $\mathcal{V}$ be the set of edges and vertices of $\mathcal{T}$, respectively. The set of all internal edges (resp. boundary 
edges) of $\mathcal{E}$ is denoted by $\mathcal{E}(\Omega)$ (resp. $\mathcal{E}(\partial \Omega)$ ). Denote the set of internal vertices (resp. vertices on the boundary) of $\mathcal{T}$ by $\mathcal{V}(\Omega)$ (resp. $\mathcal{V}(\partial \Omega)$ ). The nonconforming Morley finite element space $\mathrm{M}(\mathcal{T})$ is defined by

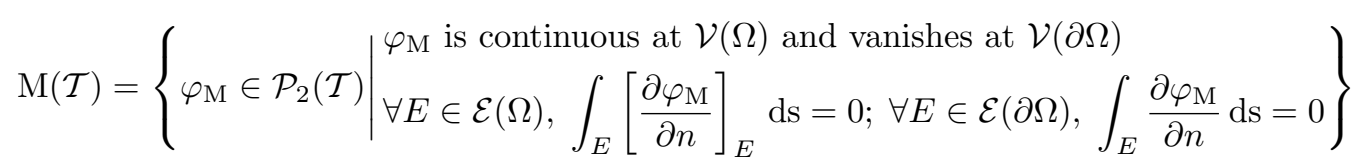

where $n$ denotes the unit outward normal to the boundary $\partial \Omega$ of $\Omega$ and $\left[\varphi_{\mathrm{M}}\right]_{E}$ is the jump of $\varphi_{\mathrm{M}}$ across any interior edge $E$. Let the Morley element space $\mathrm{M}(\mathcal{T})$ be equipped with the piecewise energy norm $\|\bullet\|_{\text {pw }}$ defined by $\left\|\varphi_{\mathrm{M}}\right\|_{\mathrm{pw}}^{2}:=\sum_{T \in \mathcal{T}}\left\|D_{\mathrm{pw}}^{2} \varphi_{\mathrm{M}}\right\|_{L^{2}(T)}^{2}$ for any $\varphi_{\mathrm{M}} \in \mathrm{M}(\mathcal{T})$, where for $j=0,1,2$; let $D_{\mathrm{pw}}^{j}$ be defined as $D_{\mathrm{pw}}^{0} \varphi_{\mathrm{M}}=\varphi_{\mathrm{M}}, D_{\mathrm{pw}}^{1} \varphi_{\mathrm{M}}=\nabla_{\mathrm{pw}} \varphi_{\mathrm{M}}$, and $D_{\mathrm{pw}}^{2} \bullet$ is the piecewise Hessian. Given the obstacle $\chi \in H^{2}(\Omega)$ with $\max \chi(\partial \Omega)<0$, define the discrete analogue [7]

$$
K(\chi, \mathcal{T}):=\left\{\varphi_{\mathrm{M}} \in \mathrm{M}(\mathcal{T}) \mid \chi(p) \leq \varphi_{\mathrm{M}}(p) \text { for all } p \in \mathcal{V}\right\}
$$

to $K$. The Morley nonconforming FEM for $(1.2)$ seeks $\left(u_{\mathrm{M}}, v_{\mathrm{M}}\right) \in K(\chi, \mathcal{T}) \times \mathrm{M}(\mathcal{T})$ such that

$$
\begin{array}{ll}
a_{\mathrm{pw}}\left(u_{\mathrm{M}}, u_{\mathrm{M}}-\varphi_{1}\right)+2 b_{\mathrm{pw}}\left(u_{\mathrm{M}}, u_{\mathrm{M}}-\varphi_{1}, v_{\mathrm{M}}\right) \leq\left(f, u_{\mathrm{M}}-\varphi_{1}\right)_{L^{2}(\Omega)} & \text { for all } \varphi_{1} \in K(\chi, \mathcal{T}), \\
a_{\mathrm{pw}}\left(v_{\mathrm{M}}, \varphi_{2}\right)-b_{\mathrm{pw}}\left(u_{\mathrm{M}}, u_{\mathrm{M}}, \varphi_{2}\right)=0 \quad \text { for all } \varphi_{2} \in \mathrm{M}(\mathcal{T}) . &
\end{array}
$$

Here and throughout this paper, for all $\eta_{\mathrm{M}}, w_{\mathrm{M}}, \varphi_{\mathrm{M}} \in \mathrm{M}(\mathcal{T})$, define

$$
\begin{aligned}
& a_{\mathrm{pw}}\left(\eta_{\mathrm{M}}, \varphi_{\mathrm{M}}\right):=\int_{\Omega} D_{\mathrm{pw}}^{2} \eta_{\mathrm{M}}: D_{\mathrm{pw}}^{2} \varphi_{\mathrm{M}} \mathrm{dx}, \\
& b_{\mathrm{pw}}\left(\eta_{\mathrm{M}}, w_{\mathrm{M}}, \varphi_{\mathrm{M}}\right):=-\frac{1}{2} \int_{\Omega}\left[\eta_{\mathrm{M}}, w_{\mathrm{M}}\right]_{\mathrm{pw}} \varphi_{\mathrm{M}} \mathrm{dx} .
\end{aligned}
$$

Note that $b_{\text {pw }}(\bullet, \bullet, \bullet)$ is symmetric with respect to the first two arguments.

Lemma 4.1 (Morley interpolation [11,15,19]). The Morley interpolation $I_{\mathrm{M}}: H_{0}^{2}(\Omega) \rightarrow \mathrm{M}(\mathcal{T})$ is defined, for $\varphi \in H_{0}^{2}(\Omega)$, by (the degrees of freedom for the Morley finite element)

$$
\left(I_{\mathrm{M}} \varphi\right)(z)=\varphi(z) \text { for any } z \in \mathcal{V} \text { and } \int_{E} \frac{\partial I_{\mathrm{M}} \varphi}{\partial n_{E}} \mathrm{ds}=\int_{E} \frac{\partial \varphi}{\partial n_{E}} \mathrm{ds} \text { for any edge } E \in \mathcal{E},
$$

and satisfies (a)-(c) for all $\psi \in H^{2}(T), T \in \mathcal{T}$, and all $\varphi \in H_{0}^{2}(\Omega) \cap H^{2+\alpha}(\Omega)$.

(a) (integral mean property of the Hessian) $D_{\mathrm{pw}}^{2} I_{\mathrm{M}}=\Pi_{0} D_{\mathrm{pw}}^{2}$ in $H_{0}^{2}(\Omega)$,

(b) (approximation and stability)

$$
\left\|h_{T}^{-2}\left(1-I_{\mathrm{M}}\right) \psi\right\|_{L^{2}(T)}+\left\|h_{T}^{-1} D_{\mathrm{pw}}\left(1-I_{\mathrm{M}}\right) \psi\right\|_{L^{2}(T)} \lesssim\left\|D_{\mathrm{pw}}^{2}\left(1-I_{\mathrm{M}}\right) \psi\right\|_{L^{2}(T)},
$$

(c) $\left\|D_{\mathrm{pw}}^{2}\left(1-I_{\mathrm{M}}\right) \varphi\right\|_{L^{2}(\Omega)} \lesssim h_{\max }^{\alpha}\|\varphi\|_{2+\alpha}$.

Lemma 4.2 (enrichment/conforming companion [15,19]). There exists a linear operator $E_{\mathrm{M}}: \mathrm{M}(\mathcal{T}) \rightarrow H_{0}^{2}(\Omega)$ such that any $\varphi_{\mathrm{M}} \in \mathrm{M}(\mathcal{T})$ satisfies $(a)-(d)$ with a universal constant $\Lambda$ that depends on the shape-regularity of $\mathcal{T}$ but not on the mesh-size $h_{\mathcal{T}}$.
(a) $I_{\mathrm{M}} E_{\mathrm{M}} \varphi_{\mathrm{M}}=\varphi_{\mathrm{M}}$,
(b) $\Pi_{0}\left(\varphi_{\mathrm{M}}-E_{\mathrm{M}} \varphi_{\mathrm{M}}\right)=0$,
(c) $\Pi_{0} D_{\mathrm{pw}}^{2}\left(\varphi_{\mathrm{M}}-E_{\mathrm{M}} \varphi_{\mathrm{M}}\right)=0$,
(d) $\sum_{j=0}^{2}\left\|h_{\mathcal{T}}^{j-2} D_{\mathrm{pw}}^{j}\left(\varphi_{\mathrm{M}}-E_{\mathrm{M}} \varphi_{\mathrm{M}}\right)\right\|_{L^{2}(\Omega)} \leq \Lambda \min _{\varphi \in H_{0}^{2}(\Omega)}\left\|D_{\mathrm{pw}}^{2}\left(\varphi_{\mathrm{M}}-\varphi\right)\right\|_{L^{2}(\Omega)}$.

Remark 4.3. Lemmas 4.1 and 4.2 lead for all $\varphi_{\mathrm{M}}, w_{\mathrm{M}}, \psi_{\mathrm{M}} \in \mathrm{M}(\mathcal{T})$ and $\psi \in H_{0}^{2}(\Omega)$ to

$$
a_{\mathrm{pw}}\left(\varphi_{\mathrm{M}}, E_{\mathrm{M}} \psi_{\mathrm{M}}-\psi_{\mathrm{M}}\right)=a_{\mathrm{pw}}\left(\varphi_{\mathrm{M}}, E_{\mathrm{M}} I_{\mathrm{M}} \psi-\psi\right)=b_{\mathrm{pw}}\left(\varphi_{\mathrm{M}}, w_{\mathrm{M}}, E_{\mathrm{M}} \psi_{\mathrm{M}}-\psi_{\mathrm{M}}\right)=0 .
$$


Lemma 4.4 (bounds for $a_{\mathrm{pw}}(\bullet, \bullet)\left[7\right.$, Lemmas 4.2 and 4.3]). Any $\varphi \in H^{2+\alpha}(\Omega), \psi \in H_{0}^{2}(\Omega) \cap H^{2+\alpha}(\Omega)$, $\psi_{\mathrm{M}}, \varphi_{\mathrm{M}} \in \mathrm{M}(\mathcal{T})$ satisfy $(a)-(c)$.

(a) $a_{\mathrm{pw}}\left(\varphi, E_{\mathrm{M}} \psi_{\mathrm{M}}-\psi_{\mathrm{M}}\right) \lesssim h_{\max }^{\alpha}\|\varphi\|_{2+\alpha}\left\|\psi_{\mathrm{M}}\right\|_{\mathrm{pw}}$,

(b) $a_{\mathrm{pw}}\left(\varphi, I_{\mathrm{M}} \psi-\psi\right) \lesssim h_{\max }^{2 \alpha}\|\varphi\|_{2+\alpha}\|\psi\|_{2+\alpha}$,

(c) the scalar product $a_{\mathrm{pw}}(\bullet, \bullet)$ is elliptic in the sense that $a_{\mathrm{pw}}\left(\varphi_{\mathrm{M}}, \varphi_{\mathrm{M}}\right)=\left\|\varphi_{\mathrm{M}}\right\|_{\mathrm{pw}}^{2}$.

Recall from (2.1), the Sobolev (resp. Friedrichs) constant $C_{\mathrm{S}}$ in the Sobolev embedding $H_{0}^{2}(\Omega) \hookrightarrow C(\bar{\Omega})$ (resp. $C_{\mathrm{F}}$ in $\left.H_{0}^{2}(\Omega) \hookrightarrow L^{2}(\Omega)\right)$. Recall the index $\alpha^{\prime}$ of elliptic regularity.

Theorem 4.5 (discrete Sobolev and Friedrichs inequalities). For $0<\alpha^{\prime}<1$, set $\beta=\alpha^{\prime}$ and for $1 \leq \alpha^{\prime}$ and any $0<\epsilon<1$, set $\beta=1-\epsilon$. Then there exist positive constants $C(\beta)$, and $C\left(\alpha^{\prime}\right)$ such that $C_{\mathrm{dS}}:=C_{\mathrm{S}}+C(\beta) h_{\max }^{\beta}$ and $C_{\mathrm{dF}}:=C_{\mathrm{F}}+C\left(\alpha^{\prime}\right) h_{\max }^{\alpha^{\prime}}$ satisfy for any $v+v_{\mathrm{M}} \in H_{0}^{2}(\Omega)+\mathrm{M}(\mathcal{T})$

$$
(a)\left\|v+v_{\mathrm{M}}\right\|_{L^{\infty}(\Omega)} \leq C_{\mathrm{dS}}\left\|v+v_{\mathrm{M}}\right\|_{\mathrm{pw}} \quad \text { and } \quad(b)\left\|v+v_{\mathrm{M}}\right\|_{L^{2}(\Omega)} \leq C_{\mathrm{dF}}\left\|v+v_{\mathrm{M}}\right\|_{\mathrm{pw}} .
$$

Proof. The point of the theorem is to get sharp estimates of $C_{\mathrm{dS}}$ and $C_{\mathrm{dF}}$, otherwise this result is a direct consequence of e.g. [13, Lemma 4.7].

(a) The piecewise uniformly continuous function $v+v_{\mathrm{M}}$ has a maximum norm that is the supremum of all integrals $\int_{\Omega}\left(v+v_{\mathrm{M}}\right) \varphi \mathrm{dx}$ for $\varphi \in L^{1}(\Omega)$ with $\|\varphi\|_{L^{1}(\Omega)}=1$. Given $\varphi \in L^{1}(\Omega)$ with $\|\varphi\|_{L^{1}(\Omega)}=1$, let $z \in H_{0}^{2}(\Omega)$ solve

$$
a(z, \bullet)=\langle\varphi, \bullet\rangle_{L^{1}(\Omega)},
$$

where the duality $\langle\bullet, \bullet\rangle_{L^{1}(\Omega)}$ extends the $L^{2}$ scalar product. For any $0<\epsilon<1$, the embedding $H^{1+\epsilon}(\Omega) \hookrightarrow$ $L^{\infty}(\Omega)$ is continuous. This implies $\langle\varphi, \bullet\rangle_{L^{1}(\Omega)} \in H^{-(1+\epsilon)}(\Omega)$. For $0<\alpha^{\prime}<1$, choose $0<\epsilon<1$ such that $0<\alpha^{\prime}<1-\epsilon$, set $\beta=\alpha^{\prime}$. For $1 \leq \alpha^{\prime}$ and any $0<\epsilon<1$, set $\beta=1-\epsilon$. The shift theorem [1, Thm. 8] in elliptic regularity shows $z \in H^{2+\beta}(\Omega)$. With bound $C(\beta, \Omega)$ of the embedding $H^{1+\epsilon}(\Omega) \hookrightarrow L^{\infty}(\Omega)$ and since $\|\varphi\|_{L^{1}(\Omega)}=1$,

$$
\|z\|_{2+\beta} \lesssim\left\|\langle\varphi, \bullet\rangle_{L^{1}(\Omega)}\right\|_{H^{-(1+\epsilon)}}=\sup _{0 \neq \psi \in H_{0}^{1+\epsilon}(\Omega)} \frac{(\varphi, \psi)_{L^{2}(\Omega)}}{\|\psi\|_{L^{\infty}(\Omega)}} \frac{\|\psi\|_{L^{\infty}(\Omega)}}{\|\psi\|_{1+\epsilon}} \leq C(\beta, \Omega) .
$$

Given $v \in H_{0}^{2}(\Omega)$ and $v_{\mathrm{M}} \in \mathrm{M}(\mathcal{T})$, let $w \in H_{0}^{2}(\Omega)$ solve $a_{\mathrm{pw}}(w, \bullet)=a_{\mathrm{pw}}\left(v+v_{\mathrm{M}}, \bullet\right) \in H^{-2}(\Omega)$. Set $\delta:=w-v-v_{\mathrm{M}}$ and recall $a_{\mathrm{pw}}(\delta, z)=0$. This, $\|\varphi\|_{L^{1}(\Omega)}=1$, and the Sobolev constant $C_{\mathrm{S}}$ lead to

$$
\begin{aligned}
\left(v+v_{\mathrm{M}}, \varphi\right)_{L^{2}(\Omega)} & =(w, \varphi)_{L^{2}(\Omega)}-(\delta, \varphi)_{L^{2}(\Omega)} \leq\|w\|_{L^{\infty}(\Omega)}-(\delta, \varphi)_{L^{2}(\Omega)} \\
& \leq C_{\mathrm{S}}\|w\|-(\delta, \varphi)_{L^{2}(\Omega)} \leq C_{\mathrm{S}}\left\|v+v_{\mathrm{M}}\right\|_{\mathrm{pw}}-(\delta, \varphi)_{L^{2}(\Omega)}
\end{aligned}
$$

Since $w-v-E_{\mathrm{M}} v_{\mathrm{M}} \in H_{0}^{2}(\Omega),(4.3)$, the Hölder inequality, $a_{\mathrm{pw}}(\delta, z)=0$, Lemma $4.2 \mathrm{c}-\mathrm{d}$, the inverse estimate, Lemma 4.1c, and (4.4) read

$$
\begin{aligned}
(\delta, \varphi)_{L^{2}(\Omega)} & \leq a\left(z, w-v-E_{\mathrm{M}} v_{\mathrm{M}}\right)+\|\varphi\|_{L^{1}(\Omega)}\left\|E_{\mathrm{M}} v_{\mathrm{M}}-v_{\mathrm{M}}\right\|_{L^{\infty}(\Omega)} \\
& \leq a_{\mathrm{pw}}\left(z-I_{\mathrm{M}} z, v_{\mathrm{M}}-E_{\mathrm{M}} v_{\mathrm{M}}\right)+C_{\mathrm{inv}}\left\|h_{\mathcal{T}}^{-1}\left(v_{\mathrm{M}}-E_{\mathrm{M}} v_{\mathrm{M}}\right)\right\|_{L^{2}(\Omega)} \\
& \lesssim\left\|v+v_{\mathrm{M}}\right\|_{\mathrm{pw}}\left(h_{\mathrm{max}}^{\beta}\|z\|_{2+\beta}+C_{\mathrm{inv}} \Lambda h_{\max }\right) \lesssim h_{\max }^{\beta}\left\|v+v_{\mathrm{M}}\right\|_{\mathrm{pw}} .
\end{aligned}
$$

The combination of the last and second-last displayed inequalities conclude the proof of $(a)$ with the constant $C_{\mathrm{S}}+C(\beta) h_{\max }^{\beta}$.

(b) Given any $\varphi \in L^{2}(\Omega)$ with $\|\varphi\|_{L^{2}(\Omega)}=1$, let $z \in H_{0}^{2}(\Omega)$ solve $a(z, \bullet)=(\varphi, \bullet)_{L^{2}(\Omega)} \in L^{2}(\Omega)$. Then, $z \in H^{2+\alpha^{\prime}}(\Omega)[4$, Thm. 2]. Note that

$$
\left\|v+v_{\mathrm{M}}\right\|_{L^{2}(\Omega)}=\sup _{\substack{\varphi \in L^{2}(\Omega) \\\|\varphi\|_{L^{2}(\Omega)}=1}}\left(v+v_{\mathrm{M}}, \varphi\right)_{L^{2}(\Omega)} .
$$


Analogous arguments of part $(a)$ apply with $\|w\|_{L^{2}(\Omega)} \leq C_{\mathrm{F}}\|w\|$ and replace $L^{\infty}(\Omega)$ and $\beta$ by $L^{2}(\Omega)$ and $\min \left\{\alpha^{\prime}, 4\right\}$, respectively. This concludes the proof of $(b)$ with the constant $C_{\mathrm{F}}+C\left(\alpha^{\prime}\right) h_{\max }^{\alpha^{\prime}}$.

Lemma 4.6 (bounds for $b_{\mathrm{pw}}(\bullet, \bullet, \bullet)\left[14\right.$, Lemma 2.6]). Any $\eta, \varphi, \phi \in H_{0}^{2}(\Omega)+\mathrm{M}(\mathcal{T})$ satisfy

$$
b_{\mathrm{pw}}(\eta, \varphi, \phi) \leq C_{\mathrm{dS}}\|\eta\|_{\mathrm{pw}}\|\varphi\|_{\mathrm{pw}}\|\phi\|_{\mathrm{pw}} .
$$

\subsection{Existence, uniqueness, and a priori bound of the discrete solution}

This section establishes the well-posedness of the discrete problem (4.1). The discrete analogue $G_{\mathrm{M}}: \mathrm{M}(\mathcal{T}) \rightarrow$ $\mathrm{M}(\mathcal{T})$ of $G$ in $(2.2)$ is characterised by $G_{\mathrm{M}}\left(\phi_{\mathrm{M}}\right) \in \mathrm{M}(\mathcal{T})$ and

$$
a_{\mathrm{pw}}\left(G_{\mathrm{M}}\left(\varphi_{\mathrm{M}}\right), \psi_{\mathrm{M}}\right)=b_{\mathrm{pw}}\left(\varphi_{\mathrm{M}}, \varphi_{\mathrm{M}}, \psi_{\mathrm{M}}\right) \quad \text { for all } \varphi_{\mathrm{M}}, \psi_{\mathrm{M}} \in \mathrm{M}(\mathcal{T}) .
$$

This gives rise to the energy functional $j_{\mathrm{pw}}(\bullet): K(\chi, \mathcal{T}) \rightarrow \mathbb{R}$ defined for all $\xi_{\mathrm{M}} \in K(\chi, \mathcal{T})$ by

$$
j_{\mathrm{pw}}\left(\xi_{\mathrm{M}}\right):=\frac{1}{2}\left\|\xi_{\mathrm{M}}\right\|_{\mathrm{pw}}^{2}+\frac{1}{2}\left\|G_{\mathrm{M}}\left(\xi_{\mathrm{M}}\right)\right\|_{\mathrm{pw}}^{2}-\left(f, \xi_{\mathrm{M}}\right)_{L^{2}(\Omega)} .
$$

Theorem 4.7 (existence, a priori and uniqueness condition). Given $(f, \chi) \in L^{2}(\Omega) \times H^{2}(\Omega)$ with $\max \chi(\partial \Omega)<0$, there exists a minimizer $u_{\mathrm{M}} \in K(\chi, \mathcal{T})$ of $j_{\mathrm{pw}}(\bullet)$; each minimizer $u_{\mathrm{M}}$ and $v_{\mathrm{M}}:=G_{\mathrm{M}}\left(u_{\mathrm{M}}\right)$ solve (4.1). There is a positive constant $C_{\mathrm{d}}(\chi)$ that depends only on $\chi$ such that any solution $\left(u_{\mathrm{M}}, v_{\mathrm{M}}\right)$ to $(4.1)$ satisfies $(a)-(b)$.

(a) $\frac{1}{2}\left\|u_{\mathrm{M}}\right\|_{\mathrm{pw}}^{2}+\left\|v_{\mathrm{M}}\right\|_{\mathrm{pw}}^{2} \leq N_{\mathrm{d}}^{2}\left(f, u_{\mathrm{M}}\right):=2 j_{\mathrm{pw}}\left(u_{\mathrm{M}}\right)+2 C_{\mathrm{dF}}^{2}\|f\|_{L^{2}(\Omega)}^{2}$

$$
\leq M_{\mathrm{d}}^{2}(f, \chi):=C_{\mathrm{d}}(\chi)+3 C_{\mathrm{dF}}^{2}\|f\|_{L^{2}(\Omega)}^{2} .
$$

(b) If $\frac{C_{\mathrm{dS}}^{2}}{4}\left\|u_{\mathrm{M}}\right\|^{2}+C_{\mathrm{dS}}\left\|v_{\mathrm{M}}\right\|<\frac{1}{2}$, then $\left(u_{\mathrm{M}}, v_{\mathrm{M}}\right)$ is the only solution to (4.1).

Proof. Step 1 shows that there exists a minimizer of $j_{\mathrm{pw}}(\bullet)$ in $K(\chi, \mathcal{T})$. The arguments of the first part of the proof of Theorem 2.2 show that there exists a sequence $\left(u_{\mathrm{M}, n}\right)_{n \in \mathbb{N}}$ in $K(\chi, \mathcal{T})$ such that $j_{\mathrm{pw}}\left(u_{\mathrm{M}, n}\right) \rightarrow$ $\beta:=\inf _{\xi_{\mathrm{M}} \in K(\chi, \mathcal{T})} j_{\mathrm{pw}}\left(\xi_{\mathrm{M}}\right) \in \mathbb{R}$ and

$$
\left\|u_{\mathrm{M}, n}\right\|_{\mathrm{pw}}^{2}+2\left\|G_{\mathrm{M}}\left(u_{\mathrm{M}, n}\right)\right\|_{\mathrm{pw}}^{2} \leq 4 j_{\mathrm{pw}}\left(u_{\mathrm{M}, n}\right)+4 C_{\mathrm{dF}}^{2}\|f\|_{L^{2}(\Omega)}^{2} .
$$

Since $\left(j_{\mathrm{pw}}\left(u_{\mathrm{M}, n}\right)\right)_{n \in \mathbb{N}}$ is convergent, the sequences $\left(u_{\mathrm{M}, n}\right)_{n \in \mathbb{N}}$ and $\left(G\left(u_{\mathrm{M}, n}\right)\right)_{n \in \mathbb{N}}$ are bounded with respect to $\|\bullet\|_{\mathrm{pw}}$. Hence, there exist $u_{\mathrm{M}}, w_{\mathrm{M}} \in \mathrm{M}(\mathcal{T})$ and a convergent subsequence $\left(u_{\mathrm{M}, n_{k}}\right)_{k \in \mathbb{N}}$ such that

$$
\left\|u_{\mathrm{M}, n_{k}}-u_{\mathrm{M}}\right\|_{\mathrm{pw}} \rightarrow 0 \text { and }\left\|G_{\mathrm{M}}\left(u_{\mathrm{M}, n_{k}}\right)-w_{\mathrm{M}}\right\|_{\mathrm{pw}} \rightarrow 0 \text { as } k \rightarrow \infty .
$$

The non-empty closed convex set $K(\chi, \mathcal{T})$ of $\mathrm{M}(\mathcal{T})$ plus the convergence of subsequence $\left(u_{\mathrm{M}, n_{k}}\right)_{k \in \mathbb{N}}$ imply $u_{\mathrm{M}} \in K(\chi, \mathcal{T})$. The definition of $G_{\mathrm{M}}(\bullet), u_{\mathrm{M}, n_{k}} \rightarrow u_{\mathrm{M}}$, and the continuity of $G_{\mathrm{M}}(\bullet)$, imply convergence of $a_{\mathrm{pw}}\left(G_{\mathrm{M}}\left(u_{\mathrm{M}, n_{k}}\right), G_{\mathrm{M}}\left(u_{\mathrm{M}, n_{k}}\right)\right)=b_{\mathrm{pw}}\left(u_{\mathrm{M}, n_{k}}, u_{\mathrm{M}, n_{k}}, G_{\mathrm{M}}\left(u_{\mathrm{M}, n_{k}}\right)\right)$ as $k \rightarrow \infty$ towards $b_{\mathrm{pw}}\left(u_{\mathrm{M}}, u_{\mathrm{M}}, G_{\mathrm{M}}\left(u_{\mathrm{M}}\right)\right)=$ $a_{\mathrm{pw}}\left(u_{\mathrm{M}}, u_{\mathrm{M}}\right)$. This and $u_{\mathrm{M}, n_{k}} \rightarrow u_{\mathrm{M}}$ show that $u_{\mathrm{M}}$ is a minimizer of $j_{\mathrm{pw}}(\bullet)$ in $K(\chi, \mathcal{T})$.

Step 2 shows that the minimizer $u_{\mathrm{M}}$ defines a solution $\left(u_{\mathrm{M}}, G_{\mathrm{M}}\left(u_{\mathrm{M}}\right)\right)$ to (4.1). Since $u_{\mathrm{M}}$ is a minimiser of $j_{\mathrm{pw}}(\bullet)$, any $\phi_{\mathrm{M}}=\varphi_{\mathrm{M}}-u_{\mathrm{M}}$ with $\varphi_{\mathrm{M}} \in K(\chi, \mathcal{T})$ satisfies

$$
D j_{\mathrm{pw}}\left(u_{\mathrm{M}}\right)\left(\phi_{\mathrm{M}}\right)=\lim _{t \rightarrow 0} \frac{j_{\mathrm{pw}}\left(u_{\mathrm{M}}+t \phi_{\mathrm{M}}\right)-j_{\mathrm{pw}}\left(u_{\mathrm{M}}\right)}{t} \geq 0 .
$$

The definitions of $j_{\mathrm{pw}}(\bullet)$ and $G_{\mathrm{M}}(\bullet)$, the symmetry of $a_{\mathrm{pw}}(\bullet, \bullet)$, and the symmetry of $b_{\mathrm{pw}}(\bullet, \bullet, \bullet)$ with respect to first and second variables lead to

$$
\frac{j_{\mathrm{pw}}\left(u_{\mathrm{M}}+t \phi_{\mathrm{M}}\right)-j_{\mathrm{pw}}\left(u_{\mathrm{M}}\right)}{t}=a_{\mathrm{pw}}\left(u_{\mathrm{M}}, \phi_{\mathrm{M}}\right)+b_{\mathrm{pw}}\left(u_{\mathrm{M}}, \phi_{\mathrm{M}}, G_{\mathrm{M}}\left(u_{\mathrm{M}}+t \phi_{\mathrm{M}}\right)\right)-\left(f, \phi_{\mathrm{M}}\right)_{L^{2}(\Omega)}
$$




$$
\begin{aligned}
& +\frac{1}{2} \frac{b_{\mathrm{pw}}\left(u_{\mathrm{M}}, u_{\mathrm{M}}, G_{\mathrm{M}}\left(u_{\mathrm{M}}+t \phi_{\mathrm{M}}\right)-G_{\mathrm{M}}\left(u_{\mathrm{M}}\right)\right)}{t} \\
& +\frac{t}{2}\left(a_{\mathrm{pw}}\left(\phi_{\mathrm{M}}, \phi_{\mathrm{M}}\right)+b_{\mathrm{pw}}\left(\phi_{\mathrm{M}}, \phi_{\mathrm{M}}, G_{\mathrm{M}}\left(u_{\mathrm{M}}+t \phi_{\mathrm{M}}\right)\right)\right) .
\end{aligned}
$$

The definition of $G_{\mathrm{M}}(\bullet)$ and the symmetry of $a_{\mathrm{pw}}(\bullet, \bullet)$ show that

$$
\begin{aligned}
b_{\mathrm{pw}}\left(u_{\mathrm{M}}, u_{\mathrm{M}}, G_{\mathrm{M}}\left(u_{\mathrm{M}}+t \phi_{\mathrm{M}}\right)-\right. & \left.G_{\mathrm{M}}\left(u_{\mathrm{M}}\right)\right)=a_{\mathrm{pw}}\left(G_{\mathrm{M}}\left(u_{\mathrm{M}}\right), G_{\mathrm{M}}\left(u_{\mathrm{M}}+t \phi_{\mathrm{M}}\right)\right)-b_{\mathrm{pw}}\left(u_{\mathrm{M}}, u_{\mathrm{M}}, G_{\mathrm{M}}\left(u_{\mathrm{M}}\right)\right) \\
& =b_{\mathrm{pw}}\left(u_{\mathrm{M}}+t \phi_{\mathrm{M}}, u_{\mathrm{M}}+t \phi_{\mathrm{M}}, G_{\mathrm{M}}\left(u_{\mathrm{M}}\right)\right)-b_{\mathrm{pw}}\left(u_{\mathrm{M}}, u_{\mathrm{M}}, G_{\mathrm{M}}\left(u_{\mathrm{M}}\right)\right) \\
& =2 t b_{\mathrm{pw}}\left(u_{\mathrm{M}}, \phi_{\mathrm{M}}, G_{\mathrm{M}}\left(u_{\mathrm{M}}\right)\right)+t^{2} b_{\mathrm{pw}}\left(\phi_{\mathrm{M}}, \phi_{\mathrm{M}}, G_{\mathrm{M}}\left(u_{\mathrm{M}}\right)\right)
\end{aligned}
$$

with the symmetry of $b_{\mathrm{pw}}(\bullet, \bullet, \bullet)$ in the first two variables in the last step. A substitution of the above identity in (4.6) leads to

$$
\begin{aligned}
\frac{j_{\mathrm{pw}}\left(u_{\mathrm{M}}+t \phi_{\mathrm{M}}\right)-j_{\mathrm{pw}}\left(u_{\mathrm{M}}\right)}{t}= & a_{\mathrm{pw}}\left(u_{\mathrm{M}}, \phi_{\mathrm{M}}\right)+b_{\mathrm{pw}}\left(u_{\mathrm{M}}, \phi_{\mathrm{M}}, G_{\mathrm{M}}\left(u_{\mathrm{M}}\right)+G_{\mathrm{M}}\left(u_{\mathrm{M}}+t \phi_{\mathrm{M}}\right)\right)-\left(f, \phi_{\mathrm{M}}\right) \\
& +\frac{t}{2}\left(a_{\mathrm{pw}}\left(\phi_{\mathrm{M}}, \phi_{\mathrm{M}}\right)+b_{\mathrm{pw}}\left(\phi_{\mathrm{M}}, \phi_{\mathrm{M}}, G_{\mathrm{M}}\left(u_{\mathrm{M}}\right)+G_{\mathrm{M}}\left(u_{\mathrm{M}}+t \phi_{\mathrm{M}}\right)\right)\right) .
\end{aligned}
$$

This, the continuity of $G_{\mathrm{M}}(\bullet)$ and $(4.5)$ show that the minimizer $u_{\mathrm{M}}$ of $j_{\mathrm{pw}}(\bullet)$ in $K(\chi, \mathcal{T})$ and $v_{\mathrm{M}}:=G_{\mathrm{M}}\left(u_{\mathrm{M}}\right)$ defines a solution $\left(u_{\mathrm{M}}, v_{\mathrm{M}}\right)$ to $(4.1)$.

Step 3 of the proof establishes bounds for the discrete solution (the proof of $(a)$ ). Since $u_{\mathrm{M}}$ is the global minimizer of $j_{\mathrm{pw}}(\bullet)$, the Young inequality, and a rearrangement of terms imply for any $\varphi_{\mathrm{M}} \in K(\chi, \mathcal{T})$ that

$$
\left\|u_{\mathrm{M}}\right\|_{\mathrm{pw}}^{2}+\left\|v_{\mathrm{M}}\right\|_{\mathrm{pw}}^{2} \leq 2 j_{\mathrm{pw}}\left(\varphi_{\mathrm{M}}\right)+2\left(f, u_{\mathrm{M}}\right)_{L^{2}(\Omega)} \leq 2 j_{\mathrm{pw}}\left(\varphi_{\mathrm{M}}\right)+2 C_{\mathrm{dF}}^{2}\|f\|_{L^{2}(\Omega)}^{2}+\frac{1}{2}\left\|u_{\mathrm{M}}\right\|^{2} .
$$

This implies

$$
\frac{1}{2}\left\|u_{\mathrm{M}}\right\|_{\mathrm{pw}}^{2}+\left\|v_{\mathrm{M}}\right\|_{\mathrm{pw}}^{2} \leq 2 j_{\mathrm{pw}}\left(\varphi_{\mathrm{M}}\right)+2 C_{\mathrm{dF}}^{2}\|f\|_{L^{2}(\Omega)}^{2}=: N_{\mathrm{d}}^{2}\left(f, \varphi_{\mathrm{M}}\right) .
$$

Given $\psi$ and $\varphi:=\chi \psi$ from the proof of Theorem 2.3, $\varphi_{\mathrm{M}}:=I_{\mathrm{M}}(\varphi) \in K(\chi, \mathcal{T})$. The properties of Morley interpolation Lemma 4.1a, definition of $G_{\mathrm{M}}(\bullet)$, the bounds of $b_{\mathrm{pw}}(\bullet, \bullet, \bullet)$ and Lemma 4.6 lead to

$$
\left\|I_{\mathrm{M}}(\varphi)\right\|_{\mathrm{pw}} \leq\|\varphi\| \text { and }\left\|G_{\mathrm{M}}\left(I_{\mathrm{M}}(\varphi)\right)\right\|_{\mathrm{pw}} \leq C_{\mathrm{dS}}\|\varphi\|^{2}
$$

The combination of above inequalities, the bound of $\|\varphi\|$ from $(2.6)$ conclude $(a)$ with $C_{\mathrm{d}}(\chi):=$ $2 C^{2}(\psi)\|\chi\|_{H^{2}\left(\Omega_{+}\right)}^{2}+C_{\mathrm{dS}}^{2} C^{4}(\psi)\|\chi\|_{H^{2}\left(\Omega_{+}\right)}^{4}$ and $M_{\mathrm{d}}^{2}(f, \chi):=C_{\mathrm{d}}(\chi)+3 C_{\mathrm{dF}}^{2}\|f\|_{L^{2}(\Omega)}^{2}$. The a priori bound in the equation (4.7) and $u_{\mathrm{M}}$ being the minimizer of $j_{\mathrm{pw}}(\bullet)$ imply $N_{\mathrm{d}}\left(f, u_{\mathrm{M}}\right) \leq N_{\mathrm{d}}\left(f, I_{\mathrm{M}}(\varphi)\right) \leq M_{\mathrm{d}}(f, \chi)$.

Step 4 establishes uniqueness of the discrete solution (the proof of (b)). Let $\left(u_{\mathrm{M}}^{j}, v_{\mathrm{M}}^{j}\right) \in K(\chi, \mathcal{T}) \times$ $\mathrm{M}(\mathcal{T})$ solve $(4.1)$ for $j=1,2$ and define $e:=u_{\mathrm{M}}^{1}-u_{\mathrm{M}}^{2}$ and $\delta:=v_{\mathrm{M}}^{1}-v_{\mathrm{M}}^{2}$. The test functions $u_{\mathrm{M}}^{2}$ (resp. $\left.u_{\mathrm{M}}^{1}\right)$ in (4.1a) and $\delta$ in (4.1b), and a simplification lead to (2.7) and (2.8) with $\|\bullet\|, b(\bullet, \bullet, \bullet), u_{1}, u_{2}, v_{1}, v_{2}$ replaced by $\|\bullet\|_{\mathrm{pw}}, b_{\mathrm{pw}}(\bullet, \bullet, \bullet), u_{\mathrm{M}}^{1}, u_{\mathrm{M}}^{2}, v_{\mathrm{M}}^{1}, v_{\mathrm{M}}^{2}$. With this substitution, the algebra in (2.7)-(2.9) holds verbatim with the further substitution of $C_{\mathrm{S}}$ and $M(f, \chi)$ by $C_{\mathrm{dS}}$ and $M_{\mathrm{d}}(f, \chi)$. Further details are omitted to conclude $e=0=\delta$ for uniqueness.

Remark 4.8 (a priori and a posteriori criteria for discrete uniqueness). The a priori smallness assumption on the data $C_{\mathrm{dS}} M_{\mathrm{d}}(f, \chi)<1 / 2$ implies the a posteriori smallness assumption $C_{\mathrm{dS}} N_{\mathrm{d}}\left(f, u_{\mathrm{M}}\right)<1 / 2$ and so the uniqueness of the solution to (4.1). (The proof is a discrete analog to Remark 2.4 and hence omitted.) 


\section{A priori ERROR ANALYSIS}

This section establishes an a priori error estimates of Morley FEM for the von Kármán obstacle problem with small data.

\subsection{Main result}

Recall $M(f, \chi)$ and $M_{\mathrm{d}}(f, \chi)$ from Theorems 2.3 and 4.7, the Sobolev and Friedrichs (resp. its discrete versions) constants $C_{\mathrm{S}}$ and $C_{\mathrm{F}}$ (resp. $C_{\mathrm{dS}}$ and $\left.C_{\mathrm{dF}}\right)$ from $(2.1)$ and Theorem 4.5, and $\beta, \alpha, C(\beta)$, and $C(\alpha)$ from Theorem 4.5. The following theorem establishes for small data an a priori energy norm error estimates that is quasi-optimal plus linear convergence.

Theorem 5.1 (energy norm estimates). For a given $f \in L^{2}(\Omega), \chi \in C^{2}(\Omega)$ with $\max \chi(\partial \Omega)<0$ and $C_{\mathrm{S}} M(f, \chi)<1 / 2$, there exists a unique solution $(u, v) \in\left(C^{2}(\Omega) \cap H^{2+\alpha}(\Omega)\right) \times\left(C^{2}(\bar{\Omega}) \cap H^{2+\alpha}(\Omega)\right)$ to $(1.2)$ and positive $\epsilon, C$ such that for any $\mathcal{T} \in \mathbb{T}(\epsilon)$ with maximal mesh size $h_{\max }$ the solution $\left(u_{\mathrm{M}}, v_{\mathrm{M}}\right) \in K(\chi, \mathcal{T}) \times M(\mathcal{T})$ to (4.1) is unique and satisfies

$$
\left\|u-u_{\mathrm{M}}\right\|_{\mathrm{pw}}+\left\|v-v_{\mathrm{M}}\right\|_{\mathrm{pw}} \leq C\left(\left\|u-I_{\mathrm{M}} u\right\|_{\mathrm{pw}}+\left\|v-I_{\mathrm{M}} v\right\|_{\mathrm{pw}}+h_{\mathrm{max}}\right) .
$$

\subsection{A priori error analysis of a shifted biharmonic obstacle problem}

Let $(u, v)$ be a solution to $(1.2)$ with the regularity $u, v \in C^{2}(\Omega) \cap H^{2+\alpha}(\Omega) \cap H_{0}^{2}(\Omega)$ from Theorem 3.5. The Sobolev embedding $H^{2+\alpha}(\Omega) \hookrightarrow W^{2,4}(\Omega)$ leads to $\tilde{f}:=f+[u, v] \in L^{2}(\Omega)$. The transformed problem seeks $u_{L} \in K$ such that

$$
a\left(u_{L}, u_{L}-\varphi\right) \leq\left(\tilde{f}, u_{L}-\varphi\right)_{L^{2}(\Omega)} \quad \text { for all } \varphi \in K
$$

Equivalently, $u_{L}$ is a minimizer in $K$ for the energy functional $J_{T}: H_{0}^{2}(\Omega) \rightarrow \mathbb{R}$, defined by $J_{T}(\xi):=\frac{1}{2} a(\xi, \xi)-$ $(\widetilde{f}, \xi)_{L^{2}(\Omega)}$ for all $H_{0}^{2}(\Omega)$,

$$
J_{T}\left(u_{L}\right)=\min _{\xi \in K} J_{T}(\xi)
$$

By construction, the solution $u$ to $(1.2 \mathrm{a})$ also solves (5.1), then uniqueness implies $u_{L}=u$.

Recall the auxiliary problem from [8] which is a continuous problem with discrete obstacle constraints. Let $K_{A}:=\left\{\xi \in H_{0}^{2}(\Omega): \forall p \in \mathcal{V}, \xi(p) \geq \chi(p)\right\}$ for the set $\mathcal{V}$ of all vertices in the triangulation $\mathcal{T}$. The biharmonic obstacle problem problem with discrete constraints seeks $u_{A} \in K_{A}$ such that

$$
a\left(u_{A}, u_{A}-\varphi\right) \leq\left(\widetilde{f}, u_{A}-\varphi\right)_{L^{2}(\Omega)} \quad \text { for all } \varphi \in K_{A} .
$$

Equivalently, $u_{A}$ is a minimizer for energy functional $J_{T}: H_{0}^{2}(\Omega) \rightarrow \mathbb{R}$ over set $K_{A}$,

$$
J_{T}\left(u_{A}\right)=\min _{\xi \in K_{A}} J_{T}(\xi) \text { for all } \xi \in K_{A}
$$

The solution $u_{L}=u$ to the biharmonic problem (5.1) and the solution $u_{A}$ to the corresponding auxiliary problem (5.3) satisfy the following result.

Lemma 5.2 (convergence rates $[8]$ ). Let $\chi \in C^{2}(\Omega) \cap C^{0}(\bar{\Omega})$ with $\max \chi(\partial \Omega)<0$, and let $u \in C^{2}(\Omega) \cap H^{2+\alpha}(\Omega)$ solve (5.1). Then there exist $\epsilon>0$ and $\widehat{u}_{A} \in K$ such that $\left\|u-u_{A}\right\| \lesssim h_{\max }\|u\|_{2+\alpha}$ and $\left\|\widehat{u}_{A}-u_{A}\right\| \lesssim h_{\max }^{2}\|u\|_{2+\alpha}$ for any triangulation $\mathcal{T} \in \mathbb{T}(\epsilon)$ with maximal mesh size $h_{\max }$ and the solution $u_{A} \in K_{A}$ to (5.3). 


\subsection{Proof of the main result}

Proof. Step 1 of the proof involves a choice of a bound for the discretization parameter for which the smallness assumption of Remark 2.4 and the uniqueness of solutions to (1.2), (4.1) hold. Define $\mu:=C_{\mathrm{S}} M(f, \chi)<1 / 2$ and its discrete analogs $\mu_{d}:=\left(C_{\mathrm{S}}+C(\beta) h_{\max }^{\beta}\right) M_{\mathrm{d}}(f, \chi)$ and $\mu_{e}:=\left(C_{\mathrm{S}}+C(\beta) h_{\max }^{\beta}\right) M(f, \chi)$ in the notation of Theorem 4.5. Since $\mu_{e}-\mu=C(\beta) h_{\max }^{\beta} M(f, \chi) \rightarrow 0$ as $h_{\max } \rightarrow 0, \mu<1 / 2$ leads to a positive constant $\epsilon_{1}$ with $\mu_{e}:=C_{\mathrm{dS}} M(f, \chi)<1 / 2$ for all $h_{\max }<\epsilon_{1}$. Theorems $2.3,4.7$, and 4.5 imply

$$
M_{\mathrm{d}}^{2}(f, \chi)-M^{2}(f, \chi)=C(\beta) h_{\max }^{\beta}\left(2 C_{\mathrm{S}}+C(\beta) h_{\max }^{\beta}\right) C^{4}(\psi)\|\chi\|_{H^{2}\left(\Omega_{+}\right)}^{4}+3 C(\alpha) h_{\max }^{\alpha}\|f\|_{L^{2}(\Omega)}
$$

and $M_{\mathrm{d}}^{2}(f, \chi)-M^{2}(f, \chi) \rightarrow 0$ as $h_{\max } \rightarrow 0$. Therefore, as $h_{\max } \rightarrow 0$,

$$
\mu_{d}^{2}-\mu^{2}=\left(\mu_{d}^{2}-\mu_{e}^{2}\right)+\left(\mu_{e}^{2}-\mu^{2}\right)=\left(C_{\mathrm{S}}+C(\beta) h_{\max }^{\beta}\right)^{2}\left(M_{\mathrm{d}}^{2}(f, \chi)-M^{2}(f, \chi)\right)+\left(\mu_{e}^{2}-\mu^{2}\right) \rightarrow 0
$$

and there exists a positive $\epsilon_{2}$ such that $\mu_{d}:=\left(C_{\mathrm{S}}+C(\beta) h_{\max }^{\beta}\right) M_{\mathrm{d}}(f, \chi)<1 / 2$ for all $h_{\max }<\epsilon_{2}$. Then $\epsilon:=\min \left\{\epsilon_{1}, \epsilon_{2}\right\}$ leads to $\mu, \mu_{d}, \mu_{e}<1 / 2$ for any $\mathcal{T} \in \mathbb{T}(\epsilon)$.

The later steps of the proof focus on the error estimates for triangulations $\mathcal{T} \in \mathbb{T}(\epsilon)$ with a unique discrete solution $\left(u_{\mathrm{M}}, v_{\mathrm{M}}\right)$ to (4.1) by Theorem 4.7. Set $e:=u-u_{\mathrm{M}}, \delta:=v-v_{\mathrm{M}}$, and the best approximation error RHS $:=\left\|u-I_{\mathrm{M}} u\right\|_{\mathrm{pw}}+\left\|v-I_{\mathrm{M}} v\right\|_{\mathrm{pw}}+h_{\max }$.

Step 2 of the proof utilizes elementary algebra to identify two critical terms

$$
T_{1}:=a\left(u, E_{\mathrm{M}} I_{\mathrm{M}} e\right)+2 a\left(v, E_{\mathrm{M}} I_{\mathrm{M}} \delta\right) \text { and } T_{2}:=-\left(a_{\mathrm{pw}}\left(u_{\mathrm{M}}, I_{\mathrm{M}} e\right)+2 a_{\mathrm{pw}}\left(v_{\mathrm{M}}, I_{\mathrm{M}} \delta\right)\right) .
$$

The definition of $a_{\mathrm{pw}}(\bullet, \bullet)$ with elementary algebra shows

$$
\|e\|_{\mathrm{pw}}^{2}=a_{\mathrm{pw}}\left(e, u-I_{\mathrm{M}} u\right)+a_{\mathrm{pw}}\left(u,\left(1-E_{\mathrm{M}}\right) I_{\mathrm{M}} e\right)+a\left(u, E_{\mathrm{M}} I_{\mathrm{M}} e\right)-a_{\mathrm{pw}}\left(u_{\mathrm{M}}, I_{\mathrm{M}} e\right) .
$$

Lemma 4.1a implies $a_{\mathrm{pw}}\left(e, u-I_{\mathrm{M}} u\right)=\left\|u-I_{\mathrm{M}} u\right\|_{\mathrm{pw}}^{2}$. The boundedness of $a_{\mathrm{pw}}(\cdot, \cdot)$, and Lemma 4.2c-d lead to

$$
a_{\mathrm{pw}}\left(u,\left(1-E_{\mathrm{M}}\right) I_{\mathrm{M}} e\right)=a_{\mathrm{pw}}\left(u-I_{\mathrm{M}} u,\left(1-E_{\mathrm{M}}\right) I_{\mathrm{M}} e\right) \leq \Lambda\left\|u-I_{\mathrm{M}} u\right\|_{\mathrm{pw}}\|e\|_{\mathrm{pw}}
$$

with $\left\|\left(1-E_{\mathrm{M}}\right) I_{\mathrm{M}} e\right\|_{\mathrm{pw}} \lesssim\left\|I_{\mathrm{M}} e\right\|_{\mathrm{pw}} \leq\|e\|_{\mathrm{pw}}$. A combination of the previous estimates leads to

$$
\|e\|_{\mathrm{pw}}^{2} \leq\left\|u-I_{\mathrm{M}} u\right\|_{\mathrm{pw}}^{2}+\Lambda\left\|u-I_{\mathrm{M}} u\right\|_{\mathrm{pw}}\|e\|_{\mathrm{pw}}+a\left(u, E_{\mathrm{M}} I_{\mathrm{M}} e\right)-a_{\mathrm{pw}}\left(u_{\mathrm{M}}, I_{\mathrm{M}} e\right) .
$$

The analogous result with $\left(u, u_{\mathrm{M}}, e\right)$ replaced by $\left(v, v_{\mathrm{M}}, \delta\right)$ reads

$$
\|\delta\|_{\mathrm{pw}}^{2} \leq\left\|v-I_{\mathrm{M}} v\right\|_{\mathrm{pw}}^{2}+\Lambda\left\|v-I_{\mathrm{M}} v\right\|_{\mathrm{pw}}\|\delta\|_{\mathrm{pw}}+a\left(v, E_{\mathrm{M}} I_{\mathrm{M}} \delta\right)-a_{\mathrm{pw}}\left(v_{\mathrm{M}}, I_{\mathrm{M}} \delta\right) .
$$

A weighted sum of those two estimates plus the Cauchy-Schwarz inequality shows

$$
\|e\|_{\mathrm{pw}}^{2}+2\|\delta\|_{\mathrm{pw}}^{2} \leq 2 \mathrm{RHS}^{2}+\Lambda\left(\left\|u-I_{\mathrm{M}} u\right\|_{\mathrm{pw}}\|e\|_{\mathrm{pw}}+2\left\|v-I_{\mathrm{M}} v\right\|_{\mathrm{pw}}\|\delta\|_{\mathrm{pw}}\right)+T_{1}+T_{2} .
$$

Step 3 of the proof employs three variational inequalities: The test function $\widehat{u}_{A} \in K$ from Lemma 5.2 leads in (1.2a) to

$$
a\left(u, u-\widehat{u}_{A}\right)+2 b\left(u, v, u-\widehat{u}_{A}\right) \leq\left(f, u-\widehat{u}_{A}\right)_{L^{2}(\Omega)} .
$$

The test function $\varphi=E_{\mathrm{M}} u_{\mathrm{M}} \in K_{A}$ and the definition of $b(\bullet, \bullet, \bullet)$ lead in (5.3) to

$$
a\left(u_{A}, u_{A}-E_{\mathrm{M}} u_{\mathrm{M}}\right) \leq\left(f, u_{A}-E_{\mathrm{M}} u_{\mathrm{M}}\right)_{L^{2}(\Omega)}-2 b\left(u, v, u_{A}-E_{\mathrm{M}} u_{\mathrm{M}}\right) .
$$

The test functions $\varphi_{1}=I_{\mathrm{M}} u \in K(\chi, \mathcal{T})$ and $\varphi_{2}=I_{\mathrm{M}} \delta \in V_{\mathrm{M}}$ lead in (4.1) to

$$
a_{\mathrm{pw}}\left(u_{\mathrm{M}}, u_{\mathrm{M}}-I_{\mathrm{M}} u\right)+2 b_{\mathrm{pw}}\left(u_{\mathrm{M}}, u_{\mathrm{M}}-I_{\mathrm{M}} u, v_{\mathrm{M}}\right) \leq\left(f, u_{\mathrm{M}}-I_{\mathrm{M}} u\right)_{L^{2}(\Omega)},
$$




$$
a_{\mathrm{pw}}\left(v_{\mathrm{M}}, I_{\mathrm{M}} \delta\right)-b_{\mathrm{pw}}\left(u_{\mathrm{M}}, u_{\mathrm{M}}, I_{\mathrm{M}} \delta\right)=0 .
$$

The sum of preceeding four displayed estimates lead to one inequality with many terms. An elementary, but lengthy algebra leads to an estimate for $\left(T_{1}+T_{2}\right) / 2$ and the crucial terms $b_{\mathrm{pw}}(e, e, v)-b_{\mathrm{pw}}(e, u, \delta)$. The following list of identities are employed in the calculation: $a_{\mathrm{pw}}\left(I_{\mathrm{M}} u, E_{\mathrm{M}} I_{\mathrm{M}} u-u\right)=b_{\mathrm{pw}}\left(u_{\mathrm{M}}, u_{\mathrm{M}}, v_{\mathrm{M}}-E_{\mathrm{M}} v_{\mathrm{M}}\right)=0$ from Remark $4.3 ; b_{\mathrm{pw}}\left(u_{\mathrm{M}}, I_{\mathrm{M}} u-u, \Pi_{0} v\right)=0$ from Lemma 4.1a where, $\Pi_{0} v \in \mathcal{P}_{0}(\mathcal{T})$ is the piecewise constant $L^{2}$ projection of $v ; b\left(u_{\mathrm{M}}, u_{\mathrm{M}}, E_{\mathrm{M}} v_{\mathrm{M}}-v_{\mathrm{M}}\right)=b_{\mathrm{pw}}\left(I_{\mathrm{M}} u, I_{\mathrm{M}} u, v_{\mathrm{M}}-E_{\mathrm{M}} v_{\mathrm{M}}\right)=0$ from Lemma $4.2 \mathrm{~b}$; $b_{\mathrm{pw}}\left(I_{\mathrm{M}} u, u_{\mathrm{M}}-E_{\mathrm{M}} u_{\mathrm{M}}, \Pi_{0} v\right)=0$ from Lemma $4.2 \mathrm{c}$; and the symmetry $b(u, u, v)=b(u, v, u)$. The resulting inequality is equivalent to

$$
\begin{aligned}
& \frac{T_{1}+T_{2}}{2}+b_{\mathrm{pw}}(e, e, v)-b_{\mathrm{pw}}(e, u, \delta) \\
& \leq \frac{1}{2} a_{\mathrm{pw}}\left(u-I_{\mathrm{M}} u, E_{\mathrm{M}} I_{\mathrm{M}} u-u\right)+a_{\mathrm{pw}}\left(v-I_{\mathrm{M}} v, E_{\mathrm{M}} I_{\mathrm{M}} v-v\right) \\
& \quad+\frac{1}{2} a\left(u, \widehat{u}_{A}-u_{A}\right)+a\left(u-u_{A}, u_{A}-E_{\mathrm{M}} u_{\mathrm{M}}\right)-b\left(u, v, u_{A}-\widehat{u}_{A}\right) \\
& \quad+b_{\mathrm{pw}}\left(u-I_{\mathrm{M}} u,\left(1-E_{\mathrm{M}}\right) u_{\mathrm{M}}, v\right)+b_{\mathrm{pw}}\left(I_{\mathrm{M}} u,\left(1-E_{\mathrm{M}}\right) u_{\mathrm{M}}, v-\Pi_{0} v\right) \\
& \quad+b_{\mathrm{pw}}\left(u-I_{\mathrm{M}} u, u,\left(1-E_{\mathrm{M}}\right) v_{\mathrm{M}}\right)+b_{\mathrm{pw}}\left(I_{\mathrm{M}} u, u-I_{\mathrm{M}} u,\left(1-E_{\mathrm{M}}\right) v_{\mathrm{M}}\right) \\
& \quad+b_{\mathrm{pw}}\left(u_{\mathrm{M}}, I_{\mathrm{M}} u-u, v_{\mathrm{M}}-v\right)+b_{\mathrm{pw}}\left(u_{\mathrm{M}}, I_{\mathrm{M}} u-u, v-\Pi_{0} v\right) \\
& \quad+b_{\mathrm{pw}}\left(u_{\mathrm{M}}, u_{\mathrm{M}}, v-I_{\mathrm{M}} v\right)+\frac{1}{2}\left(f, u-\widehat{u}_{A}+u_{A}-E_{\mathrm{M}} u_{\mathrm{M}}-I_{\mathrm{M}} e\right)_{L^{2}(\Omega)} \\
& = \\
& : T_{3}+\cdots+T_{15} .
\end{aligned}
$$

Step 4 of the proof estimates the terms $T_{3}, \ldots, T_{15}$ on the right-hand side of (5.6) and establishes the bound $C$ RHS $\left(h_{\max }+\|e\|_{\mathrm{pw}}+\|\delta\|_{\mathrm{pw}}\right)$ with a constant $C \approx 1$ that depends on $\|u\|,\|v\|,\|u\|_{2+\alpha},\|v\|_{2+\alpha},\|f\|_{L^{2}(\Omega)}, \Lambda$ and is independent of $h_{\max }$. Elementary algebra lead to first equality in

$$
T_{3}=a_{\mathrm{pw}}\left(u-I_{\mathrm{M}} u,\left(E_{\mathrm{M}}-1\right) I_{\mathrm{M}} u\right)-\left\|u-I_{\mathrm{M}} u\right\|_{\mathrm{pw}}^{2} \leq(\Lambda-1)\left\|u-I_{\mathrm{M}} u\right\|_{\mathrm{pw}}^{2},
$$

with the Cauchy-Schwarz inequality and Lemma $4.2 \mathrm{~d}$ in the final step. The analysis of $v$ replaced by $u$ as in the estimate of $T_{3}$ reads

$$
T_{4}=a_{\mathrm{pw}}\left(v-I_{\mathrm{M}} v, E_{\mathrm{M}} I_{\mathrm{M}} v-v\right) \leq(1+\Lambda)\left\|v-I_{\mathrm{M}} v\right\|_{\mathrm{pw}}^{2} .
$$

Lemma 5.2 and the Cauchy-Schwarz inequality show

$$
T_{5}+T_{7}=\frac{1}{2} a\left(u, \widehat{u}_{A}-u_{A}\right)-b\left(u, v, u_{A}-\widehat{u}_{A}\right) \lesssim h_{\max }^{2}\|u\|_{2+\alpha}\left(\|u\|+\|[u, v]\|_{L^{2}(\Omega)}\right) .
$$

The Cauchy-Schwarz inequality, a triangle inequality, Lemma 5.2, and Lemma 4.2d lead to

$$
T_{6}=a\left(u-u_{A}, u_{A}-E_{\mathrm{M}} u_{\mathrm{M}}\right) \leq\left\|u-u_{A}\right\|\left\|u_{A}-E_{\mathrm{M}} u_{\mathrm{M}}\right\| \lesssim h_{\max }\|u\|_{2+\alpha}\left(h_{\max }\|u\|_{2+\alpha}+(1+\Lambda)\|e\|_{\mathrm{pw}}\right) .
$$

The boundedness of $b_{\mathrm{pw}}(\cdot, \cdot, \cdot)$, Lemma $4.2 \mathrm{~d}$, a triangle inequality, and $\left\|v-\Pi_{0} v\right\|_{L^{\infty}(\Omega)} \lesssim h_{\max }\|v\|_{1, \infty} \lesssim$ $h_{\max }\|v\|_{2+\alpha}$ lead to

$$
\begin{aligned}
T_{8}+T_{9} & =b_{\mathrm{pw}}\left(u-I_{\mathrm{M}} u,\left(1-E_{\mathrm{M}}\right) u_{\mathrm{M}}, v\right)+b_{\mathrm{pw}}\left(I_{\mathrm{M}} u,\left(1-E_{\mathrm{M}}\right) u_{\mathrm{M}}, v-\Pi_{0} v\right) \\
& \lesssim\|e\|_{\mathrm{pw}}\left(\left\|u-I_{\mathrm{M}} u\right\|_{\mathrm{pw}}\|v\|+h_{\max }\|u\|\|v\|_{2+\alpha}\right) .
\end{aligned}
$$

Lemma $4.2 \mathrm{~d}$ and Lemma 4.6 imply

$$
T_{10}+T_{11}=b_{\mathrm{pw}}\left(u-I_{\mathrm{M}} u, u,\left(1-E_{\mathrm{M}}\right) v_{\mathrm{M}}\right)+b_{\mathrm{pw}}\left(I_{\mathrm{M}} u, u-I_{\mathrm{M}} u,\left(1-E_{\mathrm{M}}\right) v_{\mathrm{M}}\right)
$$




$$
\lesssim\|u\|\left\|u-I_{\mathrm{M}} u\right\|_{\mathrm{pw}}\|\delta\|_{\mathrm{pw}} .
$$

Lemma 4.6 and a piecewise Poincaré inequality show

$$
\begin{aligned}
T_{12}+T_{13} & =b_{\mathrm{pw}}\left(u_{\mathrm{M}}, I_{\mathrm{M}} u-u, v_{\mathrm{M}}-v\right)+b_{\mathrm{pw}}\left(u_{\mathrm{M}}, I_{\mathrm{M}} u-u, v-\Pi_{0} v\right) \\
& \lesssim\left\|u_{\mathrm{M}}\right\|_{\mathrm{pw}}\left\|u-I_{\mathrm{M}} u\right\|_{\mathrm{pw}}\left(\|\delta\|_{\mathrm{pw}}+h_{\mathrm{max}}\|v\|_{2+\alpha}\right) .
\end{aligned}
$$

Remark 4.3, Lemma 4.2a, a generalised Hölder inequality, interpolation estimate [16, (6.1.5)], and Lemma 4.2d lead to

$$
\begin{aligned}
T_{14} & =b_{\mathrm{pw}}\left(u_{\mathrm{M}}, u_{\mathrm{M}}, v-E_{\mathrm{M}} I_{\mathrm{M}} v\right)=b_{\mathrm{pw}}\left(u_{\mathrm{M}}, u_{\mathrm{M}},\left(v-E_{\mathrm{M}} I_{\mathrm{M}} v\right)-I_{\mathrm{M}}\left(v-E_{\mathrm{M}} I_{\mathrm{M}} v\right)\right) \\
& \leq\left\|u_{\mathrm{M}}\right\|_{\mathrm{pw}}^{2}\left\|\left(v-E_{\mathrm{M}} I_{\mathrm{M}} v\right)-I_{\mathrm{M}}\left(v-E_{\mathrm{M}} I_{\mathrm{M}} v\right)\right\|_{L^{\infty}(\Omega)} \\
& \lesssim h_{\mathrm{max}}\left\|u_{\mathrm{M}}\right\|_{\mathrm{pw}}^{2}\left\|v-E_{\mathrm{M}} I_{\mathrm{M}} v\right\|_{\mathrm{pw}} \lesssim h_{\max }\left\|u_{\mathrm{M}}\right\|_{\mathrm{pw}}^{2}\left\|v-I_{\mathrm{M}} v\right\|_{\mathrm{pw}} .
\end{aligned}
$$

The Cauchy-Schwarz inequality, Lemma 5.2, Lemma 4.1b-c, a triangle inequality, and Lemma 4.2d imply

$$
\begin{aligned}
T_{15}= & \left(f, u-\widehat{u}_{A}+u_{A}-E_{\mathrm{M}} u_{\mathrm{M}}-I_{\mathrm{M}} e\right)_{L^{2}(\Omega)}=\left(f, u_{A}-\widehat{u}_{A}\right)_{L^{2}(\Omega)} \\
& +\left(f,\left(1-I_{\mathrm{M}}\right)\left(u-E_{\mathrm{M}} u_{\mathrm{M}}\right)\right)_{L^{2}(\Omega)} \lesssim h_{\max }^{2}\|f\|_{L^{2}(\Omega)}\left(\|u\|_{2+\alpha}+\|e\|_{\mathrm{pw}}\right) .
\end{aligned}
$$

The summary of all the aforementioned estimates and the estimates $\left\|u-I_{\mathrm{M}} u\right\|_{\mathrm{pw}} \leq\|e\|_{\mathrm{pw}}$ and $\left\|v-I_{\mathrm{M}} v\right\|_{\mathrm{pw}} \leq$ $\|\delta\|_{\mathrm{pw}}$ show that $T_{3}+\cdots+T_{15} \lesssim \operatorname{RHS}\left(h_{\mathrm{max}}+\|e\|_{\mathrm{pw}}+\|\delta\|_{\mathrm{pw}}\right)$.

Step 5 finishes the proof. Recall $0<\mu, \mu_{e}<1 / 2$ for $\mathcal{T} \in \mathbb{T}(\epsilon)$ from Step 1 and abbreviate for $0<\gamma \leq 1$, $g(\gamma):=\frac{C_{\mathrm{dS}}^{2}}{4 \gamma}\|u\|^{2}+C_{\mathrm{S}}\|v\|$. Remark 2.4 and Theorem 4.5 imply $g(1)<\frac{1}{2}$. Since $g(\gamma)$ is continuous, there exists some $\gamma_{0}$ with $0<\gamma_{0}<1$ such that $g\left(\gamma_{0}\right)<\frac{1}{2}$. Lemma 4.6 and a weighted Young inequality in the end imply

$$
b_{\mathrm{pw}}(e, u, \delta)-b_{\mathrm{pw}}(e, e, v) \leq C_{\mathrm{dS}}\|u\|\|e\|_{\mathrm{pw}}\|\delta\|_{\mathrm{pw}}+C_{\mathrm{S}}\|e\|_{\mathrm{pw}}^{2}\|v\| \leq g\left(\gamma_{0}\right)\|e\|_{\mathrm{pw}}^{2}+\gamma_{0}\|\delta\|_{\mathrm{pw}}^{2} .
$$

The combination of this with the upper bound of $T_{3}, \cdots, T_{15}$ in (5.6) from Step 4 reads

$$
\frac{T_{1}+T_{2}}{2}-\left(g\left(\gamma_{0}\right)\|e\|_{\mathrm{pw}}^{2}+\gamma_{0}\|\delta\|_{\mathrm{pw}}^{2}\right) \leq C \operatorname{RHS}\left(h_{\mathrm{max}}+\|e\|_{\mathrm{pw}}+\|\delta\|_{\mathrm{pw}}\right)
$$

with the universal constant $C \approx 1$ that depends on $\|u\|,\|v\|,\|u\|_{2+\alpha},\|v\|_{2+\alpha},\|f\|_{L^{2}(\Omega)}, \Lambda$ and is independent of $h_{\text {max }}$. The point is that $g\left(\gamma_{0}\right)<\frac{1}{2}$ and $\gamma_{0}<1$. Multiply (5.7) by 2 and combine it with (5.5) to derive some $\varkappa \approx \min \left\{1-2 g\left(\gamma_{0}\right), 2\left(1-\gamma_{0}\right)\right\}>0$ that satisfies

$$
\varkappa\left(\|e\|_{\mathrm{pw}}^{2}+\|\delta\|_{\mathrm{pw}}^{2}\right) \leq \mathrm{RHS}^{2}+\mathrm{RHS} \sqrt{\|e\|_{\mathrm{pw}}^{2}+\|\delta\|_{\mathrm{pw}}^{2}} \leq\left(1+\frac{1}{2 \varkappa}\right) \mathrm{RHS}^{2}+\frac{\varkappa}{2}\left(\|e\|_{\mathrm{pw}}^{2}+\|\delta\|_{\mathrm{pw}}^{2}\right)
$$

with a weighted Young inequality in the last step. The last estimate concludes the proof of $\|e\|_{\mathrm{pw}}^{2}+\|\delta\|_{\mathrm{pw}}^{2} \leq$ $\varkappa^{-2}(2 \varkappa+1)$ RHS $^{2}$.

\section{Implementation Procedure and Numerical Results}

The first subsection is devoted to the implementation procedure to solve the discrete problem (4.1). Subsections 6.2 and 6.3 deal with the results of the numerical experiments and is followed by a subsection on conclusions. 


\subsection{Implementation procedure}

The solution $\left(u_{\mathrm{M}}, v_{\mathrm{M}}\right)$ to (4.1) is computed using a combination of Newtons' method [24] in an inner loop and primal dual active set strategy [21] in an outer loop. The initial value $u_{\mathrm{M}}^{\text {init }}$ for $u_{\mathrm{M}}$ in the iterative scheme is the discrete solution to the biharmonic obstacle problem: seek $u_{\mathrm{M}}^{\text {init }} \in K(\chi, \mathcal{T})$ such that

$$
a_{\mathrm{pw}}\left(u_{\mathrm{M}}^{\text {init }}, u_{\mathrm{M}}^{\text {init }}-\varphi_{\mathrm{M}}\right) \leq\left(f, u_{\mathrm{M}}^{\text {init }}-\varphi_{\mathrm{M}}\right)_{L^{2}(\Omega)} \quad \text { for all } \varphi_{\mathrm{M}} \in K(\chi, \mathcal{T})
$$

with $K(\chi, \mathcal{T})$ from Subsection 4.1. Since (6.1) is (4.1a) without the trilinear term, $u_{M}^{\text {init }}$ is computed with the same algorithm below without the inner loop for the nonlinearity. This is shown in Figure 1 and the general case is described in the sequel.

Recall $\mathrm{M}(\mathcal{T}), \mathcal{V}$ and $\mathcal{E}$ from Subsection 4.1. Let $p \in \mathcal{V}(\Omega)$ and $\left(\varphi_{1}, \ldots, \varphi_{N}\right)$ be the node- and edge-oriented basis functions in $\mathrm{M}(\mathcal{T}), N:=|\mathcal{V}(\Omega)|+|\mathcal{E}(\Omega)|$; see [11] for details and basic algorithms for the Morley FEM. Let $u_{\mathrm{M}}=\sum_{j=1}^{N} \alpha_{j} \varphi_{j}$ and $v_{\mathrm{M}}=\sum_{j=1}^{N} \beta_{j} \varphi_{j}$ with $\boldsymbol{\alpha}=\left(\alpha_{1}, \ldots, \alpha_{N}\right)$ and $\boldsymbol{\beta}=\left(\beta_{1}, \ldots, \beta_{N}\right)$.

Primal dual active set strategy.

- Choose initial values $\left(u_{\mathrm{M}}^{0}, v_{\mathrm{M}}^{0}\right)=\left(u_{\mathrm{M}}^{\text {init }}, 0\right)$.

- In the $m$ th step of the primal dual active set algorithm, find the active $A c^{m}$ and inactive $I n^{m}$ sets defined by

$$
\begin{aligned}
& A c^{m}=\left\{p \in \mathcal{V}(\Omega): \lambda^{m-1}(p)+\chi(p)-u_{\mathrm{M}}^{m-1}(p) \leq 0\right\}, \\
& I^{m}=\left\{p \in \mathcal{V}(\Omega): \lambda^{m-1}(p)+\chi(p)-u_{\mathrm{M}}^{m-1}(p)>0\right\} .
\end{aligned}
$$

Since the degrees of freedom also involve the midpoints of the interior edges, let $I^{m}:=I n^{m} \cup \mathcal{E}(\Omega)$ be the union of $I n^{m}$ and the midpoints of interior edges.

(a) Non-linear system.

* The matrix formulation corresponding to (4.1) can be expressed as block matrices in term of active and inactive sets and load vector $\mathbf{F}$ on the right-hand side.

$\star$ Impose $u_{\mathrm{M}}^{m}\left(A c^{m}\right)=\chi\left(A c^{m}\right)=\boldsymbol{\alpha}^{m}\left(A c^{m}\right)$ and $\boldsymbol{\lambda}^{m}\left(I^{m}\right)=0$. From here on, superscript $m$ is omitted and $\left(\boldsymbol{\alpha}\left(I^{m}\right), \boldsymbol{\lambda}\left(A c^{m}\right), \boldsymbol{\beta}\left(A c^{m}\right), \boldsymbol{\beta}\left(I^{m}\right)\right)$ is replaced by $\left(\boldsymbol{\alpha}_{2}, \boldsymbol{\lambda}_{1}, \boldsymbol{\beta}_{1}, \boldsymbol{\beta}_{2}\right)$ for notational convenience.

$\star$ After substitution of the known values $\boldsymbol{\alpha}(A c)$ and $\boldsymbol{\lambda}(I)=0$, the discrete problem reduces to a smaller non-linear system of equations $\mathbf{G}\left(\boldsymbol{\alpha}_{2}, \boldsymbol{\lambda}_{1}, \boldsymbol{\beta}_{1}, \boldsymbol{\beta}_{2}\right)=\mathbf{0}$.

(b) Newtons iteration with initial guess $\mathbf{S}^{0}=\left(\boldsymbol{\alpha}_{2}^{0}, \boldsymbol{\lambda}_{1}^{0}, \mathbf{0}, \mathbf{0}\right)$.

$\star$ For $\mathbf{S}^{n}:=\left(\boldsymbol{\alpha}_{2}^{n}, \boldsymbol{\lambda}_{1}^{n}, \boldsymbol{\beta}_{1}^{n}, \boldsymbol{\beta}_{2}^{n}\right)$, do $\mathbf{S}^{n+1}=\mathbf{S}^{n}-\Delta \mathbf{S}^{n}$ for the solution $\Delta \mathbf{S}^{n}$ of the linear system of equations $\mathbf{J}_{\mathbf{G}}\left(\mathbf{S}^{n}\right) \Delta \mathbf{S}^{n}=\mathbf{G}\left(\mathbf{S}^{n}\right)$ with $\mathbf{J}_{\mathbf{G}}$ is the Jacobian matrix of $\mathbf{G}$ until $\left\|\Delta \mathbf{S}^{n}\right\|_{l^{2}\left(\mathbb{R}^{2 N}\right)}$ is less than a given tolerance.

- Update $m=m+1$. This primal-dual active strategy iteration procedure terminates when $A c^{m}=A c^{m-1}$ and $I^{m}=I^{m-1}$.

The flowchart (see, Figure 1) demonstrates the combined primal-dual active set and Newton algorithms for $\mathcal{T}_{0}, \mathcal{T}_{1}, \ldots$

We observe in the examples of this paper (for small $f$ and $\chi$ ) that at each iteration of primal dual active set algorithm, the Newtons' method converges in four iterations. In this case, we notice that the error between final level and the previous level of the nodal and edge-oriented values in Euclidean norm of $\mathbb{R}^{2 N}$ is less than $10^{-9}$. Also, the primal dual active set algorithm terminates within three steps.

The uniform mesh refinement has been done by red-refinement criteria, where each triangle is subdivided into four sub-triangles by connecting the midpoints of the edges. Let $u_{\ell}$ (resp. $v_{\ell}$ ) be the discrete solution at the $\ell$ th level for $\ell=1,2,3, . ., L$ and define

$$
e_{\ell}(u):=\left\|u_{L}-u_{\ell}\right\|_{\mathrm{pw}} \text { and } \widetilde{e}_{\ell}(u):=\max _{p \in \mathcal{V}_{\ell}}\left|u_{L}(p)-u_{\ell}(p)\right| .
$$




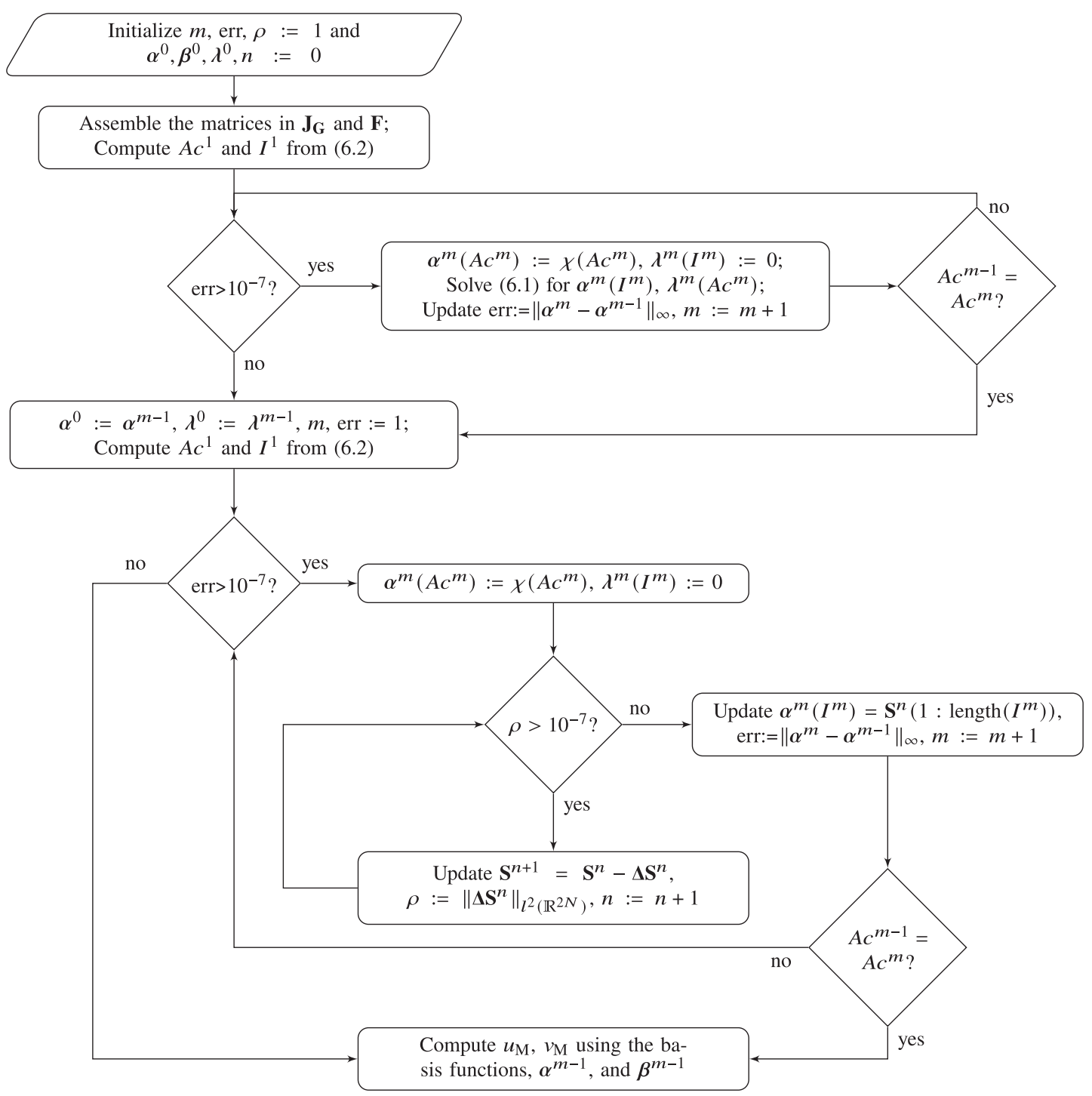

FIGURE 1. Flowchart for the primal-dual active set strategy with the Newtons method.

$$
\left(\operatorname{resp.} e_{\ell}(v):=\left\|v_{L}-v_{\ell}\right\|_{\mathrm{pw}} \text { and } \widetilde{e}_{\ell}(v):=\max _{p \in \mathcal{V}_{\ell}}\left|v_{L}(p)-v_{\ell}(p)\right|\right)
$$

The order in $H^{2}$ norm (resp. $L^{\infty}$ norm) at $\ell$ th level for $u$ is approximated by $\operatorname{EOC}(\ell):=$ $\log \left(e_{\ell}(u) / e_{L-1}(u)\right) / \log \left(2^{L-1-\ell}\right)\left(\operatorname{resp} . \log \left(\widetilde{e}_{\ell}(u) / \widetilde{e}_{L-1}(u)\right) / \log \left(2^{L-1-\ell}\right)\right)$ for $\ell=1, \ldots, L-2$. The discrete coincidence set is $\mathcal{C}_{\ell}:=\left\{p \in \mathcal{V}_{\ell} ; u_{\ell}(p)-\chi(p) \leq \widetilde{e}_{\ell}(u)\right\}$ for the level $\ell$.

Remark 6.1 (motivation for the algorithm). The standard primal dual active set strategy of [21] is employed to approximate solutions of obstacle problem governed by the linear PDE. It can be interpreted as a semismooth Newton method (for linear problems) and it converges superlinearly if the initial guess is chosen sufficiently close to the solution. For the von Kármán equations, the discrete solution is computed using the Newtons' method and it converges quadratically provided the initial guess is chosen sufficiently close to the solution $[13,24]$. 

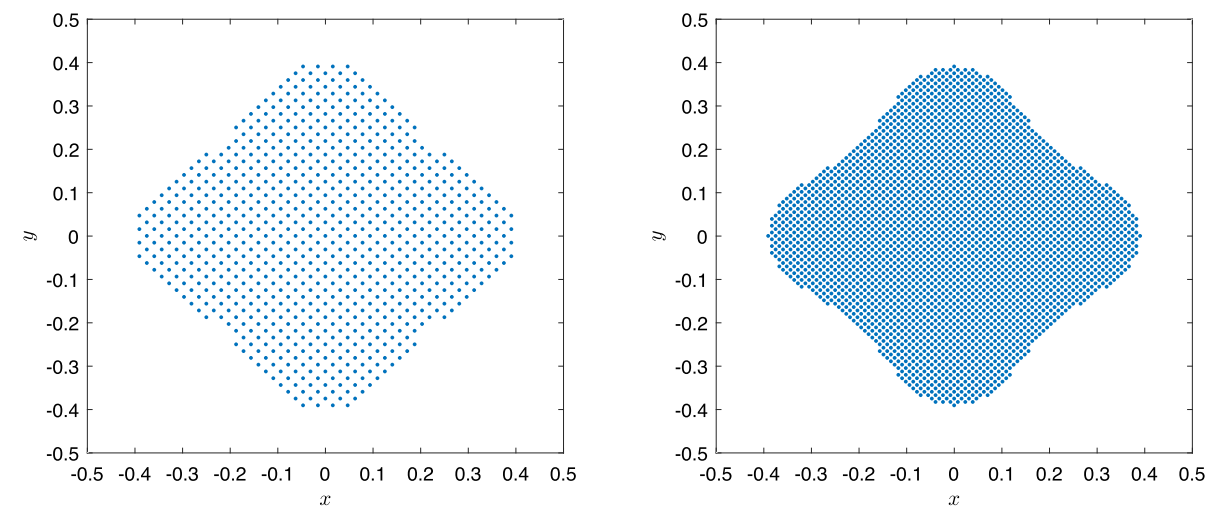

Figure 2. $\mathcal{C}_{6}$ and $\mathcal{C}_{7}$, Example 6.2.

TABLE 1. Convergence results for Example 6.2 on the square domain.

\begin{tabular}{cccccccccc}
\hline \hline$\ell$ & $h$ & $\tilde{e}_{\ell}(u)$ & $\mathrm{EOC}$ & $\tilde{e}_{\ell}(v)$ & $\mathrm{EOC}$ & $e_{\ell}(u)$ & $\mathrm{EOC}$ & $e_{\ell}(v)$ & $\mathrm{EOC}$ \\
\hline 1 & 0.5000 & 0.013222 & 1.2098 & 0.125162 & 1.9151 & 16.496069 & 0.7666 & 1.409870 & 0.9561 \\
2 & 0.2500 & 0.013222 & 1.5123 & 0.045884 & 2.0319 & 12.963642 & 0.8714 & 1.025239 & 1.0802 \\
3 & 0.1250 & 0.011327 & 1.9419 & 0.012143 & 2.0699 & 8.621491 & 0.9657 & 0.493374 & 1.0885 \\
4 & 0.0625 & 0.003404 & 2.0456 & 0.003205 & 2.1440 & 4.927900 & 1.0450 & 0.235687 & 1.0999 \\
5 & 0.0313 & 0.000909 & 2.1862 & 0.000808 & 2.3000 & 2.541191 & 1.1345 & 0.114679 & 1.1605 \\
6 & 0.0156 & 0.000200 & - & 0.000164 & - & 1.157459 & - & 0.051304 & - \\
\hline
\end{tabular}

The initial guess for this problem is chosen as the solution of the biharmonic part of f(apprvon Kármán equations, as, e.g., in [12, Section 7].

This motivates the algorithm of Figure 1 that combines Newtons' method and primal-dual active set strategy to compute the discrete solution $\left(u_{\mathrm{M}}, v_{\mathrm{M}}\right)$ to the von Kármán obstacle problem. The initial guess in this case for the loop in the Newton's method is computed by solving the biharmonic obstacle problem (as in [21]).

The algorithm proposed in Figure 1 runs successfully for all the examples in this paper. To the best of our knowledge, the proof of convergence of the algorithm of obstacle problems governed by nonlinear systems is an open question.

\subsection{The von Kármán obstacle on the square domain}

Let the computational domain be $\Omega=0.5(-1,1)^{2}$. The criss-cross mesh with $h=1$ is taken as the initial triangulation $\mathcal{T}_{0}$ of $\Omega$. Consider the von Kármán obstacle problem (1.2) for the three examples in this section. Examples 6.2 and 6.3 take $f=0$ with different obstacles; Example 6.4 concerns a significantly huge function $f$.

Example 6.2. [Coincidence set with non-zero measure]. Let the obstacle be given by $\chi(\boldsymbol{x})=1-5|\boldsymbol{x}|^{2}+|\boldsymbol{x}|^{4}, \boldsymbol{x} \in$ $\Omega=0.5(-1,1)^{2}$. This example is taken from [7]. The discrete coincidence $\mathcal{C}_{6}$ and $\mathcal{C}_{7}$ are displayed in Figure 2. Since $\Delta^{2} \chi=64>0$ in this example, it is known from [10, Section 8] that the non-coincidence set $\Omega \backslash \mathcal{C}$ is connected. This behaviour of the non-coincidence set can be seen in Figure 2 for levels 6 and 7 .

Table 1 shows errors and orders of convergence for the displacement $u$ and the Airy-stress function $v$. Observe that linear order of convergences are obtained for $u$ and $v$ in the energy norm, and quadratic order of convergence in $L^{\infty}$ norm. These numerical order of convergence in energy norm clearly matches the expected order of 

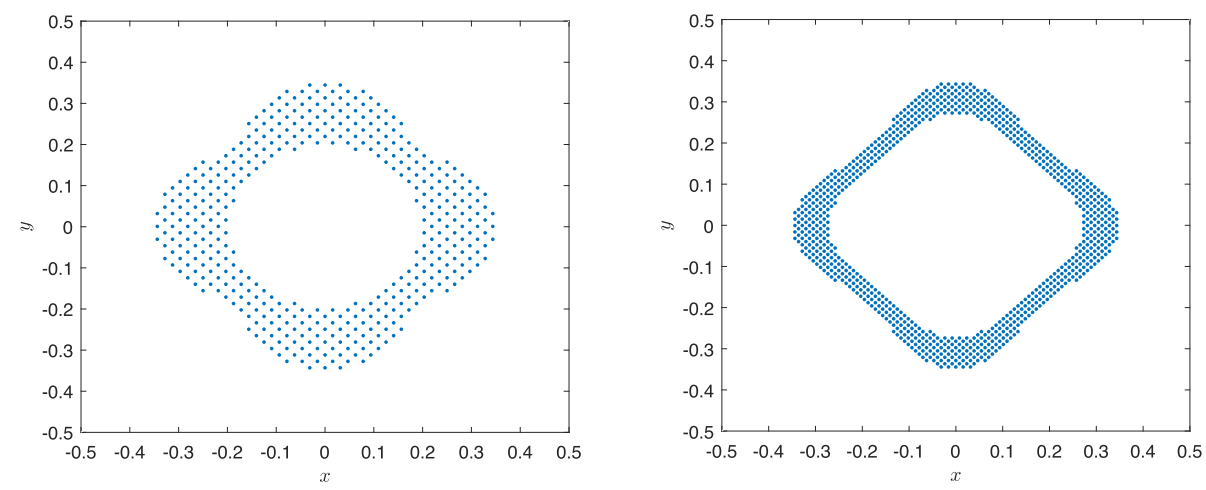

Figure 3. $\mathcal{C}_{6}$ and $\mathcal{C}_{7}$, Example 6.3.

TABLE 2. Convergence results for Example 6.3 on the square domain.

\begin{tabular}{cccccccccc}
\hline \hline$\ell$ & $h$ & $\tilde{e}_{\ell}(u)$ & $\mathrm{EOC}$ & $\tilde{e}_{\ell}(v)$ & $\mathrm{EOC}$ & $e_{\ell}(u)$ & $\mathrm{EOC}$ & $e_{\ell}(v)$ & $\mathrm{EOC}$ \\
\hline 1 & 0.5000 & 0.028792 & 1.4917 & 0.136864 & 1.8793 & 15.510398 & 0.7999 & 1.493256 & 0.9636 \\
2 & 0.2500 & 0.028792 & 1.8646 & 0.050539 & 1.9898 & 11.837363 & 0.9024 & 1.070278 & 1.0843 \\
3 & 0.1250 & 0.009347 & 1.9451 & 0.014530 & 2.0535 & 7.563740 & 0.9878 & 0.510661 & 1.0899 \\
4 & 0.0625 & 0.003116 & 2.1252 & 0.003980 & 2.1462 & 4.210097 & 1.0591 & 0.244868 & 1.1047 \\
5 & 0.0312 & 0.000843 & 2.3636 & 0.001030 & 2.3427 & 2.138703 & 1.1411 & 0.118649 & 1.1642 \\
6 & 0.0156 & 0.000164 & - & 0.000203 & - & 0.969687 & - & 0.052944 & - \\
\hline
\end{tabular}

convergence given in Theorem 5.1. Though the theoretical rate of convergence in $L^{\infty}$ norm is not analysed, the numerical rates are obtained similar to that in [7] for the biharmonic obstacle problem.

Example 6.3. [Coincidence set with zero measure] In this example taken from [7], $\chi(\boldsymbol{x})=1-5|\boldsymbol{x}|^{2}-|\boldsymbol{x}|^{4}, \boldsymbol{x} \in$ $\Omega=0.5(-1,1)^{2}$ with $\Delta^{2} \chi=-64<0$ in $\Omega$, and hence, the interior of the coincidence set must be empty, since $\Delta^{2} u$ (in the sense of distributions) is a nonnegative measure ([10, Section 8$]$ ). This can be observed in the pictures of the discrete coincidence sets displayed in Figure 3.

The errors and orders of convergence for the displacement and the Airy-stress function are presented in Table 2. The orders of convergence results are similar to those obtained in Example 6.2. Note that Examples 1 and 2 are similar except in the sign of the term $|\boldsymbol{x}|^{4}$ that appears in the obstacle function.

Example 6.4. [Violation of smallness assumption] It is interesting to observe that for $\chi$ and $\Omega$ from Example 6.2 with the source term $f=(x+3)^{2}(x-3)^{2}(y+3)^{2}(y-3)^{2}$, the primal dual active set algorithm is not convergent in 100 iterations of the algorithm. Consider $w(x, y)=(x+0.5)^{2}(y+0.5)^{2}(0.5-x)^{2}(0.5-y)^{2} \in H_{0}^{2}(\Omega)$. Then $\frac{\|w\|_{L^{2}(\Omega)}}{\|w\|}=0.0278$ and $\frac{\|w\|_{L^{\infty}(\Omega)}}{\|w\|}=0.0683$. Since $C_{\mathrm{F}}$ (resp. $C_{\mathrm{S}}$ ) is the supremum of $\frac{\|z\|_{L^{2}(\Omega)}}{\|z\|}\left(\right.$ resp. $\left.\frac{\|z\|_{L^{\infty}(\Omega)}}{\|z\|}\right)$ for all $z \in H_{0}^{2}(\Omega)$, this implies $C_{\mathrm{F}} \geq 0.0278$ (resp. $C_{\mathrm{S}} \geq 0.0683$ ). Use the definition of $M(f, \chi)$ to obtain $C_{S} M(f, \chi) \geq 20.7972$. Therefore the sufficient condition in Theorem 5.1 is violated.

For Example 6.2 with obstacle $\chi$ replaced by $\lambda \chi$, where $\lambda \in \mathbb{R}$, we noticed that the algorithm fails to converge for $\lambda \geq 4$ on $\mathcal{T}_{4}$ and $\mathcal{T}_{5}$. This illustrates the requirement of smallness assumption on the obstacle for optimal convergence rate. 

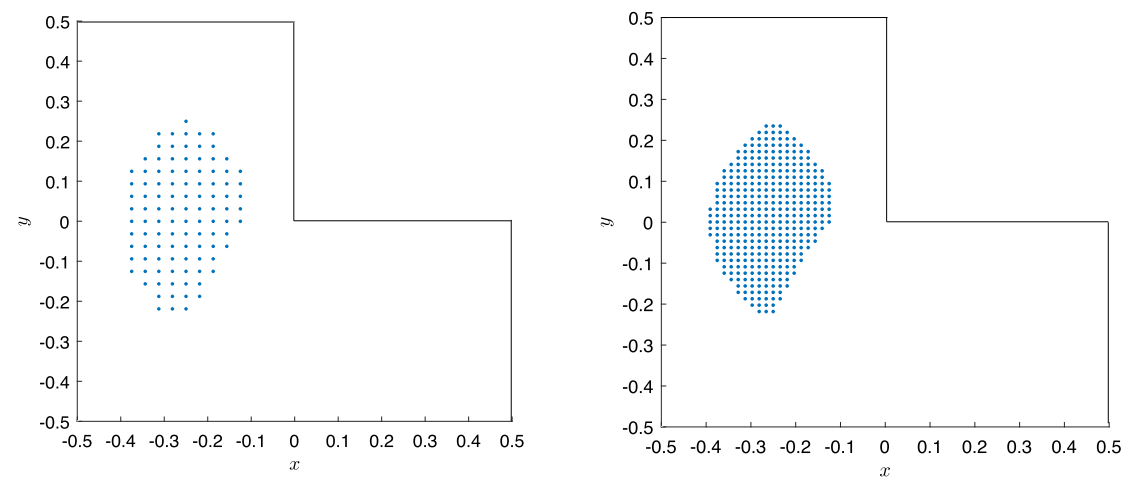

Figure $4 . \mathcal{C}_{5}$ and $\mathcal{C}_{6}$.

TABLE 3. Convergence results for the L-shaped domain.

\begin{tabular}{cccccccccc}
\hline \hline$\ell$ & $h$ & $\tilde{e}_{\ell}(u)$ & $\mathrm{EOC}$ & $\tilde{e}_{\ell}(v)$ & $\mathrm{EOC}$ & $e_{\ell}(u)$ & $\mathrm{EOC}$ & $e_{\ell}(v)$ & $\mathrm{EOC}$ \\
\hline 1 & 0.3536 & 0.046700 & 0.8276 & 0.141271 & 1.8003 & 23.203954 & 0.7177 & 2.260261 & 0.9584 \\
2 & 0.1768 & 0.021021 & 0.7196 & 0.056794 & 1.9621 & 18.313668 & 0.8431 & 1.530842 & 1.0905 \\
3 & 0.0884 & 0.025796 & 1.2271 & 0.017919 & 2.1111 & 11.746209 & 0.9442 & 0.761967 & 1.1324 \\
4 & 0.0442 & 0.014152 & 1.5879 & 0.004655 & 2.2774 & 6.556709 & 1.0473 & 0.352575 & 1.1531 \\
5 & 0.0221 & 0.004708 & - & 0.000960 & - & 3.172522 & - & 0.158538 & - \\
\hline
\end{tabular}

\subsection{The von Kármán obstacle problem on the L-shaped domain}

Consider L-shaped domain $\Omega=(-0.5,0.5)^{2} \backslash[0,0.5]^{2}, f=0$ and

$$
\chi(\boldsymbol{x})=1-\frac{(x+0.25)^{2}}{0.2^{2}}-\frac{y^{2}}{0.35^{2}}
$$

as in [7]. Choosing the initial mesh size as $h=0.7071$, the successive red-refinement algorithm computes $\mathcal{T}_{1}, \ldots, \mathcal{T}_{5}$.

Since $\Omega$ is non-convex (reduced elliptic regularity $\alpha=0.5445$, [7, Example 4]), we expect only sub-optimal order of convergences in energy norm and $L^{\infty}$ norm, that is, $\mathcal{O}\left(h^{\alpha}\right)$ convergence rate in the energy norm (see, Theorem 5.1). However, linear order of convergence is preserved in the energy norm which indicates that the numerical performance is carried out in the non-asymptotic region. The discrete coincidence sets for last two levels are depicted in Figure 4. The non-coincidence set is connected, which agrees with the result in [10] since $\Delta^{2} \chi=0$ in $\Omega$ in this example.

The convergence rates in Table 3 are not in direct contradiction to Theorem 5.1 but the reduced elliptic regularity suggests a lower rate $\alpha=0.5445$ for L-shaped domain. A similar observation is in [7, Table 5.5] with orders of convergence $\approx 0.8$ (resp. 1 ) for energy (resp. $L^{\infty}$ ) norm. In [7], the numbers are computed with the alternative definitions for error $e_{\ell}(u):=\left\|u_{\ell-1}-u_{\ell}\right\|_{\mathrm{pw}}\left(\operatorname{resp} . \widetilde{e}_{\ell}(u):=\max _{p \in \mathcal{V}_{\ell-1}}\left|u_{\ell-1}(p)-u_{\ell}(p)\right|\right)$ and order of convergence $\operatorname{EOC}(\ell):=\log \left(e_{\ell-1}(u) / e_{\ell}(u)\right) / \log (2)\left(\operatorname{resp} \cdot \log \left(\widetilde{e}_{\ell-1}(u) / \widetilde{e}_{\ell}(u)\right) / \log (2)\right)$. With these definitions, undisplayed numerical experiments confirm the numbers displayed in [7, Table 5.5] precise up to the last digit. This numerical experiment suggests that our implementation is at least consistent with the one in [7]. One possible explanation is that the corner singularity affects the asymptotic convergence rate for very small meshsizes only. This is known, for instance, for the L-shaped domain and the Poisson model problem with constant right hand side in the Courant ( $\mathcal{P}_{1}$ conforming) finite element method. The expected rate $2 / 3$ is visible only beyond $2 \times 10^{6}$ triangles with far better empirical convergence rates before that. 


\subsection{Conclusions}

The numerical results for the Morley FEM in the von Kármán obstacle problem are presented for square domain and L-shaped domain in Sections 6.2 and 6.3. The outputs obtained for the square domain confirm the theoretical rates of convergence given in Theorem 5.1 for $\alpha=1$. Example 6.4 in Section 6.3 illustrates the requirement of smallness assumption on the obstacle for optimal convergence rate. For the L-shaped domain, we expect reduced convergence rates in energy and $L^{\infty}$ norms from the elliptic regularity. However, linear order of convergence is preserved in the energy norm which indicates that the numerical performance is carried out in the non-asymptotic region.

Acknowledgements. The authors thankfully acknowledge the support from the MHRD SPARC project (ID 235) titled "The Mathematics and Computation of Plates" and the second to fifth authors also thank the hospitality of the HumboldtUniversität zu Berlin for the corresponding periods 1st June 2019-31st August 2019 (second and fifth authors), 24th June 2019-30th June 2019 (third author), and 1st July 2019-31st July 2019 (fourth author). The authors thank one anonymous referee for the constructive suggestion that led to sharper uniqueness criteria in Theorems $2.3 \mathrm{~b}$ and $4.7 \mathrm{~b}$ and to Remark 3.6.

\section{REFERENCES}

[1] C. Bacuta, J.H. Bramble and J.E. Pasciak, Shift Theorems for the Biharmonic Dirichlet Problem. In: T.F. Chan, Y. Huang, T. Tang, J. Xu, L.A. Ying (eds) Recent Progress in Computational and Applied PDES. Springer, Boston, MA (2002) 1-26.

[2] M.S. Berger and P.C. Fife, Von Kármán equations and the buckling of a thin elastic plate, II plate with general edge conditions. Commun. Pure Appl. Math. 21 (1968) 227-241.

[3] H. Blum and R. Rannacher, On mixed finite element methods in plate bending analysis. Comput. Mech. 6 (1990) $221-236$.

[4] H. Blum, R. Rannacher and R. Leis, On the boundary value problem of the biharmonic operator on domains with angular corners. Math. Methods Appl. Sci. 2 (1980) 556-581.

[5] S.C. Brenner, M. Neilan, A. Reiser and L.-Y. Sung. A $C^{0}$ interior penalty method for a von Kármán plate. Numerische Mathematik 135 (2017) 803-832.

[6] S.C. Brenner, L.-Y. Sung, H. Zhang and Y. Zhang. A quadratic $C^{0}$ interior penalty method for the displacement obstacle problem of clamped Kirchhoff plates. SIAM J. Numer. Anal. 50 (2012) 3329-3350.

[7] S.C. Brenner, L.-Y.Sung, H. Zhang and Y. Zhang, A Morley finite element method for the displacement obstacle problem of clamped Kirchhoff plates. J. Comput. Appl. Math. 254 (2013) 31-42.

[8] S.C. Brenner, L.-Y. Sung and Y. Zhang. Finite element methods for the displacement obstacle problem of clamped plates. Math. Comput. 81 (2012) 1247-1262.

[9] F. Brezzi. Finite element approximations of the von Kármán equations. ESAIM: M2AN 12 (1978) 303-312.

[10] L.A. Caffarelli and A. Friedman. The obstacle problem for the biharmonic operator. Annali della Scuola Normale Superiore di Pisa-Classe di Scienze 6 (1979) 151-184.

[11] C. Carstensen, D. Gallistl and J. Hu. A discrete Helmholtz decomposition with Morley finite element functions and the optimality of adaptive finite element schemes. Comput. Math. Appl. 68 (2014) 2167-2181.

[12] C. Carstensen, G. Mallik and N. Nataraj, A priori and a posteriori error control of discontinuous Galerkin finite element methods for the von Kármán equations. IMA J. Numer. Anal. 39 (2019) 167-200.

[13] C. Carstensen, G. Mallik and N. Nataraj, Nonconforming finite element discretization for semilinear problems with trilinear nonlinearity. IMA J. Numer. Anal. 41 (2021) 164-205.

[14] C. Carstensen and N. Nataraj, Adaptive Morley FEM for the von Kármán equations with optimal convergence rates. SIAM J. Numer. Anal. Preprint: arXiv:1908.08013 (2020).

[15] C. Carstensen and S. Puttkammer, How to prove the discrete reliability for nonconforming finite element methods. J. Comput. Math. 38 (2020) 142-175.

[16] P.G. Ciarlet (eds), The finite element method for elliptic problems, Vol. 4. Studies in Mathematics and its Applications. North-Holland Publishing Co., Amsterdam-New York-Oxford (1978).

[17] P.G. Ciarlet. Mathematical Elasticity: Volume II: Theory of Plates, Vol. 27. Studies in Mathematics and its Applications. Elsevier (1997).

[18] J. Frehse. Zum Differenzierbarkeitsproblem bei Variationsungleichungen höherer Ordnung. Abhandlungen aus dem Mathematischen Seminar der Universität Hamburg 36 (1971) 140-149.

[19] D. Gallistl. Morley finite element method for the eigenvalues of the biharmonic operator. IMA J. Numer. Anal. 35 (2014) 1779-1811.

[20] R. Glowinski. Lectures on Numerical Methods for Non-linear Variational Problems. Springer-Verlag, Berlin-Heidelberg (2008).

[21] M. Hintermüller, K. Ito and K. Kunisch, The primal-dual active set strategy as a semismooth Newton method. SIAM J. Optim. 13 (2002) 865-888. 
[22] D. Kinderlehrer and G. Stampacchia, An introduction to variational inequalities and their applications, Vol. 88. Pure and Applied Mathematics. Academic Press (1980).

[23] G.H. Knightly, An existence theorem for the von Kármán equations. Arch. Ration. Mech. Anal. 27 (1967) $233-242$.

[24] G. Mallik and N. Nataraj, Conforming finite element methods for the von Kármán equations. Adv. Comput. Math. 42 (2016) 1031-1054.

[25] G. Mallik and N. Nataraj, A nonconforming finite element approximation for the von Kármán equations. ESAIM: M2AN 50 (2016) 433-454.

[26] E. Miersemann and H.D. Mittelmann. Stability in obstacle problems for the von Kármán plate. SIAM J. Math. Anal. 23 (1992) 1099-1116.

[27] T. Miyoshi, A mixed finite element method for the solution of the von Kármán equations. Numerische Mathematik 26 (1976) 255-269.

[28] A.D. Muradova and G.E. Stavroulakis, A unilateral contact model with buckling in von Kármán plates. Nonlinear Anal.: Real World Appl. 8 (2007) 1261-1271.

[29] K. Ohtake, J.T. Oden and N. Kikuchi, Analysis of certain unilateral problems in von Kármán plate theory by a penalty method-part 1. a variational principle with penalty. Comput. Methods Appl. Mech. Eng. 24 (1980) 187-213.

[30] K. Ohtake, J.T. Oden and N. Kikuchi, Analysis of certain unilateral problems in von Kármán plate theory by a penalty method-part 2. approximation and numerical analysis. Comput. Methods Appl. Mech. Eng. 24 (1980) 317-337.

[31] A. Quarteroni, Hybrid finite element methods for the von Kármán equations. Calcolo 16 (1979) 271-288.

[32] L. Reinhart, On the numerical analysis of the von Kármán equations: mixed finite element approximation and continuation techniques. Numerische Mathematik 39 (1982) 371-404.

[33] S.-T. Yau and Y. Gao, Obstacle problem for von Kármán equations. Adv. Appl. Math. 13 (1992) $123-141$.

\section{Subscribe to Open (S2O) A fair and sustainable open access model}

This journal is currently published in open access under a Subscribe-to-Open model (S2O). S2O is a transformative model that aims to move subscription journals to open access. Open access is the free, immediate, online availability of research articles combined with the rights to use these articles fully in the digital environment. We are thankful to our subscribers and sponsors for making it possible to publish this journal in open access, free of charge for authors.

\section{Please help to maintain this journal in open access!}

Check that your library subscribes to the journal, or make a personal donation to the S2O programme, by contacting subscribers@edpsciences.org

More information, including a list of sponsors and a financial transparency report, available at: https://www. edpsciences.org/en/maths-s2o-programme 